\title{
Survivorship - lebenslange Begleitung von Krebspatienten
}

\author{
Eva-Maria Kalusche-Bontemps ${ }^{a} \quad$ Monika Reuss-Borst $^{b}$ Pia Heußner ${ }^{c}$ UIf Seifart ${ }^{d}$ \\ Georgia Schilling ${ }^{e}$ Jörn D. Beck ${ }^{f}$ Barbara Zimmer $^{g}$
}

${ }^{a}$ MediClin Reha-Zentrum Reichshof, Reichshof-Eckenhagen, Deutschland;

${ }^{b}$ Reha-Klinik Am Kurpark, Rehazentren-Baden-Württemberg, Bad Kissingen, Deutschland;

c Psycho-Onkologie Medizinische Klinik III, Klinikum der Universität München-Großhadern, München, Deutschland;

${ }^{\mathrm{d}}$ Klinik Sonnenblick, Marburg, Deutschland;

${ }^{e}$ Klinik für Tumorbiologie, Freiburg i.Br., Deutschland;

${ }^{f}$ Extraordinarius im Ruhestand, Kinder- und Jugendklinik, Universitätsklinikum Erlangen,

Comprehensive Cancer Center Erlangen - EMN, Nürnberg, Deutschland;

gKompetenz-Centrum Onkologie, Medizinischer Dienst Nordrhein, Düsseldorf, Deutschland

\section{Onkologische Rehabilitation - wer braucht welche Maßnahmen?}

\section{Referentin: Eva-Maria Kalusche-Bontemps, Reichshof-Eckenhagen, für den Arbeitskreis onkologische Rehabilitation in der DGHO}

Für Leistungen zur medizinischen Rehabilitation kommen verschiedene Kostenträger auf, wobei die Hauptlast die Deutsche Rentenversicherung (DRV) trägt. Sie führt Leistungen nach $\$ 15$ SGB VI durch mit dem Ziel, die Erwerbsfähigkeit ihrer Versicherten zu erhalten oder wiederherzustellen. Weitere Träger von Reha-Maßnahmen sind die Krankenkassen (z.B. für Nichterwerbstätige) sowie die gesetzliche Unfallversicherung. Der Gesetzgeber unterscheidet zwischen stationärer Rehabilitation sowie ganztägig ambulanter Rehabilitation, die über niedergelassene Haus- bzw. Fachärzte beantragt werden, und der Anschlussrehabilitation (AR) direkt nach einem Krankenhausaufenthalt. Darüber hinaus gibt es nach \$31 SGB VI präventive Leistungen zur Sicherung der Erwerbsfähigkeit, die onkologische Rehabilitation (altersunabhängig) sowie die stationäre Rehabilitation von Kindern und Jugendlichen.

Die DRV veröffentlich jährlich umfangreiche Statistiken zu ihren Leistungen im Bereich der medizinischen Rehabilitation, die kostenfrei im Internet eingesehen werden können [1]. Die Kürzung und Budgetierung der Rehabilitation durch das Wachstumsund Beschäftigungsförderungsgesetz (WFG) hat sich in den vergangenen Jahren deutlich auf die Zahl der Anträge, Bewilligungen und abgeschlossenen Leistungen ausgewirkt (Abb. 1).

2013 wurden von den DRV-Versicherten fast 1,7 Millionen Reha-Anträge gestellt, von denen 1,08 Millionen bewilligt wurden
[2]. Die Zahlen sind gegenüber 2012 leicht rückläufig (-1,3 bzw. -1,1\%). 2013 wurden Leistungen für Erwachsene zu 16\% aufgrund von Neubildungen durchgeführt, womit der Anteil dieser Leistungsform gegenüber dem Jahr 2000 trotz steigender Inzidenz von Krebserkrankungen nur unwesentlich zugenommen hat. Bezogen auf ausschließlich stationäre Rehabilitationen fanden diese 2013 bei Frauen zu 20\% und bei Männern zu 15\% aufgrund von Neubildungen statt, im Bereich der ambulanten Rehabilitation entfielen dagegen auf die Krebserkrankungen bei beiden Geschlechtern lediglich 2\%. Neubildungen sind bei Frauen der dritthäufigste Anlass für eine Rehabilitation (nach orthopädischen und psychischen Indikationen inklusive Sucht) und bei Männern der vierthäufigste Reha-Grund (nach Orthopädie, psychischen Erkrankungen/Sucht und Gefäßerkrankungen).

Welche Voraussetzungen für eine Rehabilitation muss der Patient mitbringen ...

Ein Patient, der sich für eine Reha-Maßnahme entschieden hat, muss «rehawillig» und «rehafähig» sein sowie eine positive Prognose bezüglich des Reha-Erfolgs haben. Es besteht eine Mitwirkungspflicht des Patienten an den Reha-Maßnahmen, wobei Mitwirkung und Motivation für den Reha-Erfolg maßgeblich sind. Der aktive Charakter einer Rehabilitation sollte dem Patienten schon vor Beginn der Maßnahme bekannt sein. Die medizinische Rehabilitation darf nicht als preiswerter Ersatz für eine geriatrische Rehabilitation oder einer Heimunterbringung verstanden werden.

... und welche die Reha-Einrichtung?

Die Reha-Einrichtungen wiederum müssen qualifizierte und spezialisierte Angebote vorhalten, am Qualitätssicherungspro-

\section{KARGER}

Fax +497614520714 
Abb. 1. Medizinische Rehabilitation: Anträge, Bewilligungen und abgeschlossene Leistungen 1991-2013. Statistik der DRV (modifiziert nach [2]).

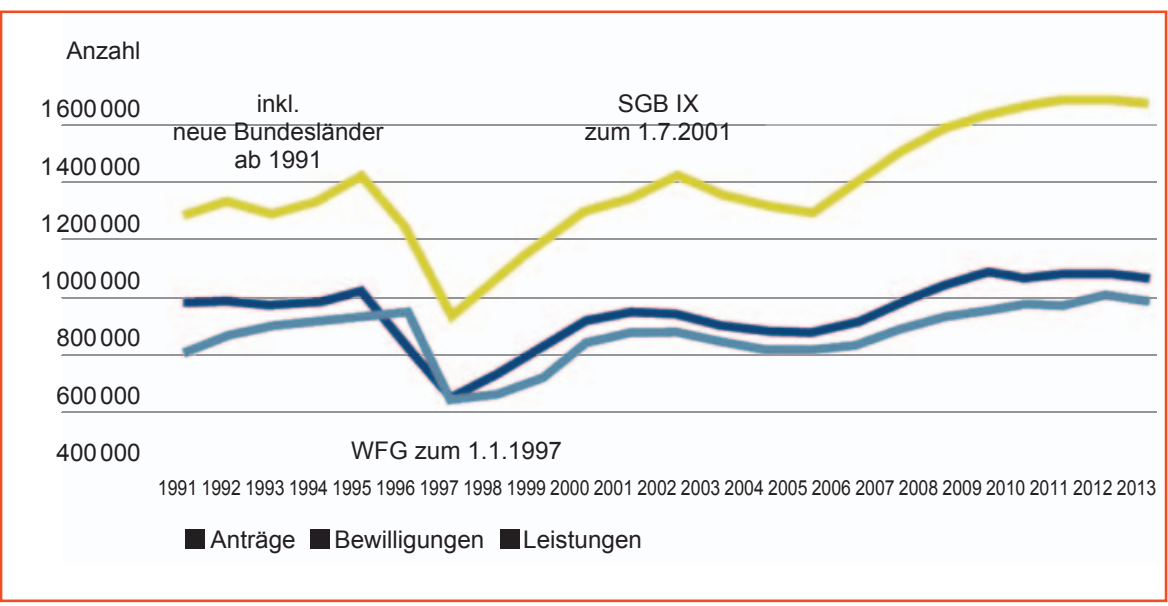

gramm der DRV teilnehmen und über eine ausreichende personelle, apparative und räumliche Ausstattung verfügen [3]. Eine ambulante Rehabilitation muss darüber hinaus den Anforderungen der Bundesarbeitsgemeinschaft für Rehabilitation e.V. (BAR) genügen.

Von den Reha-Anbietern wird ein ganzheitlicher Reha-Ansatz in Verbindung mit einem umfassenden Reha- und Therapiekonzept erwartet. Es sind klare Reha- und Therapieziele zu definieren und für jeden Patienten ein individueller Therapie- bzw. RehaPlan zu erstellen, der die Zielsetzungen der verschiedenen Therapiebereiche beschreibt und sich an einer langfristigen Strategie zur Krankheitsbewältigung orientiert.

Im Rahmen der Qualitätssicherung werden von den Kostenträgern in jährlichen Abständen umfangreiche Daten angefordert und analysiert. Sie dienen unter anderem als Diskussionsgrundlage für die konzeptionelle Ausrichtung der Kliniken. Außerdem finden seitens der Kostenträger in mehrjährigen Abständen Visitationen zur Sicherung der Strukturqualität statt.

Die Prozessqualität der rehabilitativen Versorgung wird seit 1998 über ein Peer-Review-Verfahren erfasst, das auf der anonymisierten Auswertung ärztlicher Entlassungsberichte basiert und die Regelhaftigkeit der Behandlung, die fallgerechte Definition von Behandlungszielen sowie die Plausibilität sozialmedizinischer Schlussfolgerungen überprüft. Zur Erfassung der Prozessqualität gehört auch die Erhebung und Auswertung der therapeutischen Leistungen entlang entsprechender Vorgaben (Klassifizierung therapeutischer Leistungen; KTL) [4]) sowie die Auswertung dieser KTL-Daten in Bezug auf die Frage, inwieweit die zu rehabilitierenden Patienten leitliniengerecht behandelt werden.

Die Messung der Ergebnisqualität erfolgt über Patientenbefragungen und durch Beurteilung der Beschwerdehäufigkeit. Hinzu kommt ein internes Qualitätsmanagement gemäß den Zertifizierungskriterien nach DEGEMED, ISO 9001 und anderen. All das fließt in ein Konzept zur Darstellung und Bewertung der Gesamtqualität ein, was ein gewisses Ranking der Kliniken ermöglicht und künftig bei der Vergütung und Zuweisung von Reha-Patienten stärker berücksichtigt werden soll.

Survivorship - lebenslange Begleitung von

Oncol Res Treat 2015;38(suppl 4):2-23

\section{Personelle und materielle Anforderungen an die Reha-Kliniken}

Reha-Einrichtungen müssen über ein interdisziplinär ausgerichtetes Reha-Team mit entsprechender Qualifikation verfügen. Dazu gehört Personal aus den Bereichen Medizin, Klinische Psychologie, Physiotherapie, Hydro-, Ergo- und Logopädie, Soziale Arbeit, Krankenpflege, Ernährung und Sport [5]. Die diagnostische Grundausstattung der Einrichtung sollte eine Beurteilung der Erkrankung bzw. Folgeschäden von Körperfunktionen und -strukturen ermöglichen und wird auch zur Abschätzung der arbeits- und alltagsbezogenen Leistungsfähigkeit des Reha-Patienten herangezogen (Leistungsdiagnostik). Nach Erfassung möglicher weiterer, auch psychischer, Belastungen wird der Reha- und Therapieplan mit den zugehörigen Behandlungselementen festgelegt.

\section{Ziele der stationären onkologischen Rehabilitation}

Ziel einer Reha-Maßnahme ist es, drohende oder bereits eingetretene Beeinträchtigungen im gesellschaftlichen und beruflichen Leben zu beseitigen oder zu mindern, deren mögliche Folgen abzuschwächen und einer weiteren Verschlimmerung vorzubeugen. Der zu Rehabilitierende soll (wieder) befähigt werden, eine Erwerbstätigkeit und/oder bestimmte Aktivitäten des täglichen Lebens möglichst in der Art und dem Ausmaß auszuüben, die für diesen Menschen erstrebenswert sind. Ziele in diesem Sinne sind z.B. (modifiziert nach [3]):

- Wiederherstellung und Erhaltung der Erwerbsfähigkeit,

- Planung und Einleitung weiterer Maßnahmen zur Teilhabe am Arbeitsleben (z.B. Anpassung des Arbeitsplatzes, Hilfen zur Erhaltung oder Erlangung eines Arbeitsplatzes),

- Kompensation (Entwicklung von Ersatzstrategien) bei Organverlust,

- Adaptation/Krankheitsverarbeitung sowie

- Maßnahmen zur Stabilisierung der erreichten Reha-Ziele (Kontaktaufnahme mit Sport- oder Selbsthilfegruppen etc.).

Favorisiert wird ein Reha-Ansatz, der dem biopsychosozialen Modell entlang der Internationalen Klassifikation der Funktionsfähigkeit, Behinderung und Gesundheit (ICF) der Weltgesundheits- 
Tab. 1. Reha-Ziele bezogen auf die Dimensionen «Körperfunktionen und Körperstrukturen»; modifiziert nach [3]

\section{Gastrointestinale Tumoren}

- Probleme der ausreichenden Nahrungsaufnahme, -verwertung (Malnutrition, Malassimilation)

- Ausscheidung (Stomata) bei veränderter Anatomie und organischfunktionellen Defiziten

Gynäkologische Tumoren

- OP-bedingte Schädigungen der Körperfunktionen und -strukturen wie Lymphödem, Einschränkungen der Schulter-Arm-Beweglichkeit

- Störungen der Sexualfunktion und der Psyche

Bronchialkarzinom

- Einschränkungen der Lungenfunktion, dadurch Reduzierung der körperlichen Leistungsfähigkeit

Hämatologische Systemerkrankungen

- Störungen des Immunsystems

Urogenitale Tumoren

- Auswirkungen der Therapie auf die Sexualfunktion und die Kontinenz

Tab. 2. Reha-Ziele bezogen auf die Dimensionen «Aktivität»; modifiziert nach [3]

Ausgerichtet auf die Abwendung, Beseitigung, Minderung, Verhütung der Verschlimmerung oder Milderung der Folgen bzw. der Zunahme der Beeinträchtigungen der Aktivitäten wie z.B.:

- Vermeidung/Beseitigung/Verminderung von Beeinträchtigungen des täglichen Lebens

- Verbesserung/Erhalt der Selbständigkeit der Selbstversorgung (z.B. An- und Ausziehen, Körperpflege, Haushaltsführung, Einkauf, Nahrungszubereitung und -aufnahme, Toilettenbenutzung)

- Verbesserung der Fortbewegung, Beweglichkeit (z.B. Gehen, Treppensteigen)

- Verbesserung der komplexen Aufgabenbewältigung am Arbeitsplatz (z.B. Ausdauer, Umgang mit Stress, Zeitdruck, Publikumsverkehr, Verantwortung, Schichtarbeit)

- Verbesserung des selbständigen und sachgerechten Umgangs mit Hilfsmitteln

- Optimierung der Krankheitsbewältigung (Coping)

- Verbesserung der Fähigkeit zur Freizeitgestaltung / zu sportlichen Aktivitäten

organisation (WHO) folgt $[6,7]$. Dementsprechend richten sich die Reha-Ziele und -Pläne an Faktoren der jeweiligen Person und der Umwelt aus, da sich beide auf das Gesundheitsproblem über die Dimensionen «Körperfunktionen/-strukturen», «Aktivitäten» und «Teilhabe» auswirken.

Mögliche Reha-Ziele unter Berücksichtigung dieser verschiedenen Aspekte sind in den Tabellen 1-4 dargestellt.

Welche Behandlungselemente der individuell zusammengestellte Therapieplan enthalten kann, lässt sich am Beispiel der Chemotherapie-induzierten Polyneuropathie (CIPN) aufzeigen. Als supportive Maßnahmen kommen Schmerztherapie, Neurorehabilitation (Physikalische Therapie/Elektrotherapie, Physiotherapie und Ergotherapie), Anpassung von geeignetem Schuhwerk und Orthesen in Betracht. Im Bereich der Ergotherapie hat sich ein
Tab. 3. Reha-Ziele bezogen auf die Dimensionen «Teilhabe»; modifiziert nach [3]

Die Teilhabe betrifft Fragen der Eingliederung, des Einbezogenseins oder der Beteiligung an Lebensbereichen, selbstbestimmt zu handeln oder Anerkennung zu finden

Die Ziele der Rehabilitanden sind gerichtet auf eine Verbesserung der Lebensqualität und Teilhabe in bedeutenden Lebensbereichen, z.B. in den Bereichen

- Arbeit und Beschäftigung

- Selbstversorgung

- des häuslichen Lebens (z.B. Haushaltsführung)

- des sozialen Lebens (z.B. Freizeitaktivitäten)

- der sozialen Integration/Reintegration (z.B. im Erwerbsleben)

- der sozialen Kompetenz

- der physischen/psychischen Unabhängigkeit

- der Mobilität (Benutzung von Verkehrsmitteln)

Tab. 4. Reha-Ziele bezogen auf die Dimensionen «Kontextfaktoren»; modifiziert nach [3]

Kontextfaktoren stellen den gesamten Lebenshintergrund einer Person dar

- Rahmenbedingungen, die die gesellschaftliche Teilhabe eines Menschen fördern oder behindern. Als umwelt- und personenbezogene Faktoren eines Menschen stehen die Kontextfaktoren in Wechselwirkung mit anderen Faktoren der ICF wie Körperfunktionen/-strukturen, Aktivitäten und Teilhabe

Rehabilitationsziele in diesem Sinne sind:

- Besserung des Informationstandes über die Krankheit

- Entwicklung von Strategien zum Abbau von Risikoverhalten (z.B. Rauchen, Alkoholmissbrauch, Fehlernährung, Bewegungsmangel, inadäquates Freizeitverhalten, körperliche und psychische Überforderung)

- Erlernen und Anwendung von Entspannungstechniken

- Schulung der Körperwahrnehmung

Sensibilitätstraining für Hände und Füße bewährt sowie das Üben im Umgang mit Hilfsmitteln wie verstärkte Griffe zur leichteren Nutzung von Geräten und anderes. Je nach Schweregrad der CIPN sind auch Änderungen am Arbeitsplatz anzuregen, etwa der Wechsel zu Arbeiten mit geringeren Ansprüchen an die Feinmotorik oder geringerer Verletzungsgefahr durch spitze/heiße Gegenstände oder unebene Böden. Die Beurteilung der Wegefähigkeit von Patienten mit persistierender und schwerwiegender CIPN ist ein besonderes sozialmedizinisches Problem. Als wegefähig gilt, wer gegebenenfalls unter Verwendung einer Gehhilfe (Stock, Stütze) viermal täglich etwas mehr als 500 Meter in etwas weniger als 20 min zurücklegen kann und in der Lage ist, zweimal täglich in der Hauptverkehrszeit öffentliche Verkehrsmittel zu benutzen, sofern kein Kraftfahrzeug zur Verfügung steht. Ist eine Wegefähigkeit gemäß dieser Definition nicht gegeben, besteht eine volle Erwerbsminderung [8].

Wer an neuropathischen Schädigungen leidet, die zu einer relevanten Beeinträchtigung der motorischen Funktionen führen, ist nicht in der Lage, dem Führen von Fahrzeugen der Gruppe 2 gerecht zu werden. Dabei ist zu prüfen, ob die erforderlichen Bewegungen in ausreichendem Umfang mit ausreichender Schnelligkeit 
Abb. 2. Sozialmedizinische Begutachtung zur Erwerbsfähigkeit [9].

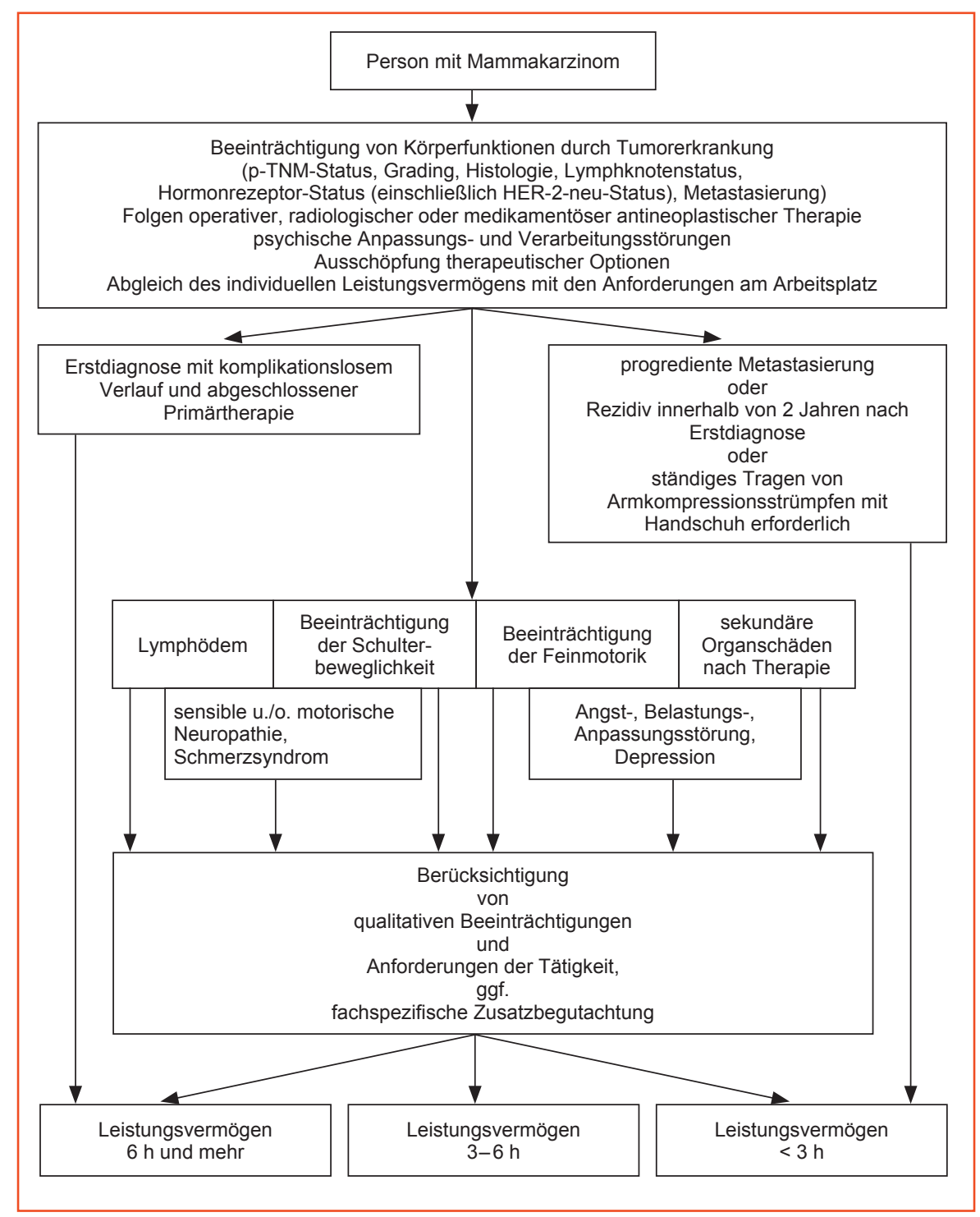

möglich sind und ob zusätzlich sensible Störungen vorliegen, die eine Beeinträchtigung der Koordination (Ataxie, Stereognosie) nach sich ziehen. Bei nicht sicher beurteilbarer Fahreignung ist eine Fahrerprobung (z.B. bei TÜV oder DEKRA) empfehlenswert.

Auch die sozialmedizinische Beurteilung der Möglichkeit zur beruflichen Wiedereingliederung ist Aufgabe der Rehabilitation und erfolgt auf Grundlage entsprechender Leitlinien. In Abbildung 2 ist der Handlungsalgorithmus zur Feststellung der Leistungsfähigkeit im Erwerbsleben bei Patientinnen mit Brustkrebs dargestellt.

\section{Mehr Forschung zur Rehabilitation}

Rehabilitation ist eine komplexe interdisziplinäre Therapieform die die Patienten wieder möglichst «fit» für ihren Alltag machen soll und ihnen eine gesunde Lebensweise vermittelt. In Deutschland müssen sich Reha-Einrichtungen zur Erfüllung dieser Aufgaben einer umfangreichen Qualitätssicherung unterziehen. Wünschenswert ist ein Ausbau der wissenschaftlichen Forschung im Bereich der Rehabilitation, um den Erfolg der Maßnahmen auf Basis von Evidenz besser als bisher belegen zu können.

\section{Onkologische Rehabilitation - was nützt sie, was ist evidenzbasiert?}

\section{Referentin: Monika Reuss-Borst, Bad Kissingen, für den Arbeitskreis onkologische Rehabilitation in der DGHO}

$\mathrm{Zu}$ den wesentlichen Zielen der onkologischen Rehabilitation gehört die Verbesserung der gesundheitsbezogenen Lebensqualität. Wir haben unter diesem Aspekt die Lebensqualität von 1879 Tumorpatienten ausgewertet, deren Rehabilitation in unserer Klinik stattfand. Zu Beginn des Aufenthalts war die Lebensqualität dieser Patienten im Vergleich zu gesunden Kontrollen gemessen am EORTC-QLQ30-Score erheblich eingeschränkt. Mit diesem validierten Messinstrument werden außer der globalen Lebensqualität auch physische, emotionale, kognitive und soziale Dimensionen der Lebensqualität sowie Fatigue erfasst. Stärker noch als die rein körperlichen Funktionen waren die emotionalen/sozialen Kompetenzen durch die Tumorerkrankung beeinträchtigt, obgleich diese 
Abb. 3. Rehabilitation in Deutschland: Das Angebot ist breitgefächert.
Onkologische Reha - was nützt sie? Was ist evidenzbasiert?

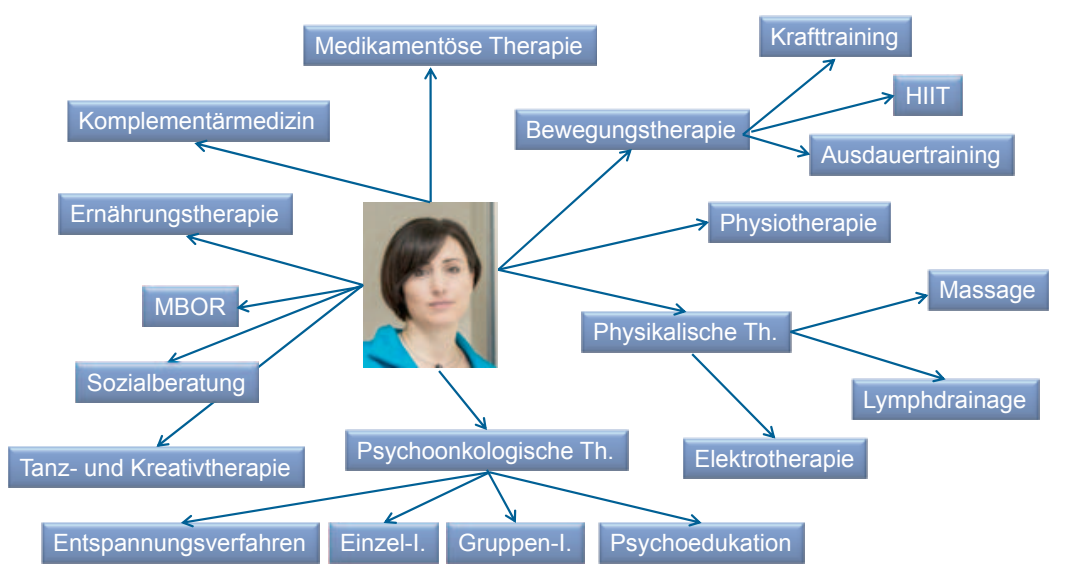

Abb. 4. Therapieziele orientieren sich an den individuellen Bedürfnissen des Patienten.
Ob eine Reha erfolgreich ist, hängt auch ab von persönlichen Ressourcen und Umweltfaktoren

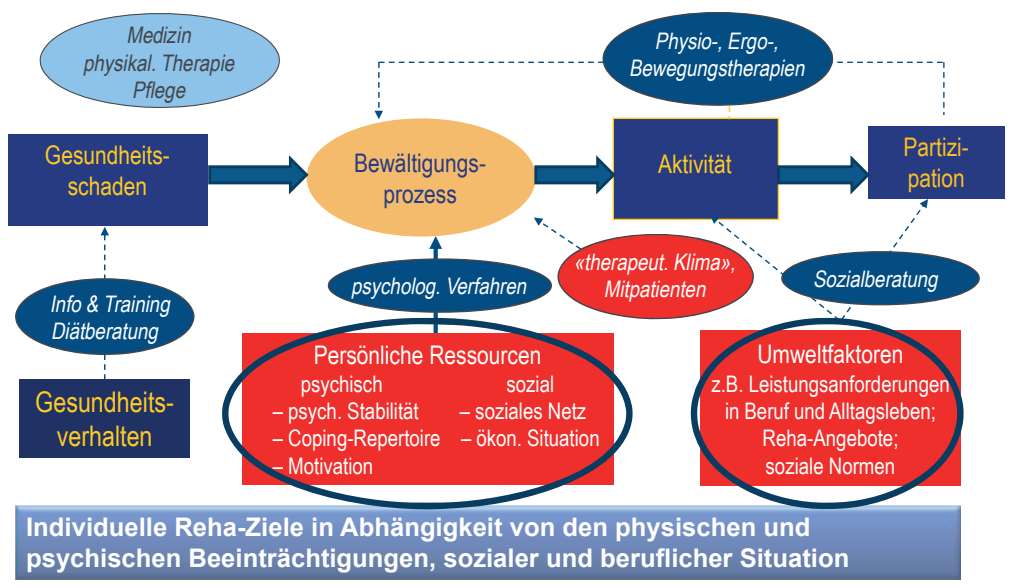

überwiegend in frühen Stadien diagnostiziert worden war. Die von uns befragten Patienten hatten somit subjektiv einen deutlichen Reha-Bedarf zum Zeitpunkt der stationären Aufnahme, die im Durchschnitt 16,3 Monate nach Erstdiagnose erfolgte. Die Rehabilitation führte zu einer signifikanten und klinisch relevanten Anhebung der Lebensqualität in allen erfassten Bereichen.

\section{Die Rehabilitation ist multimodal}

Die Reha-Konzepte in Deutschland sind multimodal angelegt (Abb. 3) und sollen den individuellen Bedarf des onkologischen Patienten beginnend mit den therapeutischen Interventionen bis hin zu der medizinisch-beruflich orientierten Rehabilitation möglichst umfassend abdecken.

Da die verschiedenen Elemente der Rehabilitation ineinandergreifen, stellt sich die Frage, was letztlich den Reha-Erfolg ausmacht: Gibt es additive oder synergistische Wirkungen, etwa im Bereich Ernährung und Bewegung? Welche Effekte haben die verschiedenen Interventionen? Welche Rolle spielen die Umgebung
(«Kurort-Effekt»), die soziale Interaktionen während der Rehabilitation sowie die Persönlichkeit des zu rehabilitierenden Menschen? Zweifellos hängt der Erfolg der Maßnahme von zahlreichen äußeren Faktoren ab (Abb. 4), die bei der Formulierung der Therapieziele zu berücksichtigen sind.

So unterschiedlich die Zielsetzung in Abhängigkeit von den persönlichen Voraussetzungen der Patienten ist, so unterschiedlich ist auch das Spektrum an Diagnosen. Mit einem Anteil von etwa 30\% stellen Frauen mit Mammakarzinom in der onkologischen Rehabilitation die größte Gruppe an Patienten dar. Reha-Patienten unterscheiden sich im Hinblick auf Prognose, Alter und Therapie-Setting (stationär, ambulant, Anschlussrehabilitation, Heilverfahren, Rehabilitation während oder regelhaft nach der Akuttherapie). Zu berücksichtigen sind auch die vorausgehenden Akuttherapien (Operation, Chemotherapie, Bestrahlung) sowie die verschiedenen Modelle der Kostenübernahme (Krankenkassen, Rentenversicherung).

Diese Heterogenität erschwert die Durchführung methodisch anspruchsvoller Studien zur Effektivität von Reha-Maßnahmen. 
Onkologische Reha - was nützt sie? Was ist evidenzbasiert?

\section{Sport- und Bewegungstherapie}

Somatische Effekte

- Kardiovaskuläre Fitness

- Körperzusammensetzung

- Muskelkraft und Muskelmasse

- Fatigue-Symptomatik

Schlafqualität

Begleiterkrankungen

- Osteoporose

- Adipositas

Arthrose

Abb. 5. Auswirkungen von Sport und Bewegung auf Befindlichkeit und Komorbidität.

Dies betrifft insbesondere die Planung und Durchführung randomisierter Placebo-kontrollierter Studien. Hervorzuheben ist auch, dass es in der Rehabilitation um komplexe Interventionen geht, deren Effekte anhand subjektiver Parameter (Fragebögen) im Sinne von «Patient-Reported Outcomes» oder mit Hilfe von Surrogatparametern erfasst werden. Hinzu kommen schwer zu kalkulierende, die Studienergebnisse beeinflussende Faktoren wie das individuelle Gesundheitsverhalten der Studienteilnehmer und deren soziale Einbettung. Auch lässt sich eine ausreichend lange Studiendauer kaum realisieren, zumal die dafür nötigen Gelder nicht aufzubringen sind.

\section{Viel Bewegung bringt viel}

Trotz dieser methodischen Schwierigkeiten gibt es eine relativ große Zahl an Studien zu nichtmedikamentösen Ansätzen in der onkologischen Rehabilitation. Besonders gut untersucht ist die Wirksamkeit einer Sport- und Bewegungstherapie. Dabei ist gut belegt, dass die körperliche Aktivität im Rahmen einer Krebserkrankung zunächst abnimmt [10]. Viele Patienten nehmen parallel dazu an Gewicht zu [11], was unter anderem mit einer Fatigue-Symptomatik, mit der spezifischen medikamentösen Behandlung (hormonell wirksame Therapien) oder auch mit einer bewusst reduzierten körperlichen Aktivität aus Angst oder Unkenntnis der Auswirkungen von Sport zusammenhängen kann. Es verwundert deshalb nicht, dass von den 1879 Teilnehmern der von uns vorgenommenen Patientenbefragung 40\% übergewichtig waren und 30\% eine Adipositas hatten. Die zahlreichen positiven Auswirkungen der Sport- und Bewegungstherapie sind gut belegt (Abb. 5). So hat die Steigerung der körperlichen Aktivität eine Reihe von somatischen Effekten, gut messbar etwa an der Zunahme der kardiovaskulären Fitness und der Verbesserung der Schlafqualität. Hinzu kommen eine Reihe günstiger psychosozialer Effekte, die zu einer besseren Krankheitsbewältigung beitragen. Sport und Bewegung wird heute schon früh in das therapeutische Angebot aufgenommen (unter Umständen bereits während der Akuttherapie), um auch die Belastung durch Komorbiditäten wie Osteoporose, Adipositas und Arthrose zu mindern. Nicht zuletzt kann Sport die Prognose der Tumorerkrankung günstig beeinflussen, wie dies insbesondere für das Mammakarzinom gezeigt worden ist, sowie - mit geringerer Evidenz - auch für das Kolon- und Prostatakarzinom.

Zusammenfassend hat sich in großen Meta-Analysen gezeigt, dass sich bei Probanden mit regelmäßiger körperlicher Bewegung die aerobe Fitness verbessert und Körpergewicht und Körperfett abnehmen [12, 13]. Auch die schon beschriebenen günstigen psychosozialen Effekte ließen sich am Beispiel Angst und Depression anhand von Meta-Analysen nachweisen [14].

Selbst ein Krafttraining, dessen Nutzen in der onkologischen Rehabilitation in der Vergangenheit eher kontrovers diskutiert wurde, ist heute kein Tabu mehr - im Gegenteil: Eine Meta-Analyse von 15 randomisiert-kontrollierten Studien unter Einbeziehung von 1652 Patientinnen mit Mammakarzinom wies nach, dass moderates Krafttraining zu einer signifikanten Reduktion der Brustkrebs-assoziierten Armlymphödeme führt sowie erwartungsgemäß zu einer signifikanten Kraftzunahme der oberen und unteren Extremitäten [15]. Die Autoren empfehlen, ein Krafttraining vermehrt in Reha-Programme aufzunehmen und dies auch in Leitlinien zu verankern.

Diese doch überzeugende Datenlage bezieht sich allerdings überwiegend auf das Mammakarzinom. Bei dieser Indikation wurden in zahlreichen Studien die Auswirkungen von Sport- und Bewegungstherapie sowohl während als auch nach der Akuttherapiephase untersucht [16]. Hinzu kommen jeweils rund ein Dutzend Studien zur Sporttherapie bei Prostatakarzinom und hämatologischen Tumoren, wogegen andere Tumorarten bisher kaum in Studien berücksichtigt worden sind.

Dass Patienten von einer körperlichen Aktivierung auch langfristig profitieren, zeigt eine hausinterne Studie. Wir haben dafür Patientinnen mit Brustkrebs verglichen, die entweder eine konventionelle, dreiwöchige Sport- und Bewegungstherapie während ihres Reha-Aufenthalts absolviert haben (Kontrollgruppe) oder an einem intensivierten Programm teilgenommen haben (Interventionsgruppe). Jedem Studienarm wurden etwa 100 Frauen zugeordnet. Patientinnen in der Kontrollgruppe behielten das empfohlene Sportprogramm (150 min moderates Ausdauertraining) auch nach Abschluss der Rehabilitation noch eine Zeit lang bei, erreichten aber nach 2 Jahren wieder das Ausgangslevel ihrer körperlichen Aktivität. Anders in der Interventionsgruppe: Diese Patientinnen wurden intensiv sportlich betreut, führten ein Trainingstagebuch und erhielten nach 4 und 8 Monaten «Refresher»-Kurse, was zu einer langfristigen Steigerung der sportlichen Betätigung führte. Auch nach 2 Jahren war der Unterschied zur Kontrollgruppe signifikant, außerdem litten die Teilnehmerinnen dieser Gruppe weniger unter Fatigue. Dies spricht unseres Erachtens für ein Aufbrechen starrer Reha-Angebote; vielmehr sollte sportlich interessierten Patienten ein auf die persönlichen Zielsetzungen abgestimmtes Sportprogramm angeboten werden.

\section{Auch psychoonkologische Interventionen gehören in die Rehabilitation}

Die Aufnahme psychoonkologischer Angebote in die Rehabilitation wird von einer ausreichend großen Datenlage gestützt, wobei die therapeutische Orientierung nicht maßgeblich zu sein 
scheint. Strukturierte psychoedukative Programme sind demnach wirksamer als andere Verfahren (etwa CBT, supportiv-expressive Therapie), wobei längere Interventionen effektiver sind als kürzere. Vorteile von Einzelsitzungen gegenüber Gruppentherapien sind nicht eindeutig belegt. Dies gilt auch für eine mögliche Verlängerung der Überlebenszeit von Tumorpatienten: Die dazu durchgeführten Studien zum Mammakarzinom kommen zu unterschiedlichen Ergebnissen, eine neuere Studie [17] konnte allerdings bei einer relativ großen Zahl von Frauen $(\mathrm{n}=227)$ bei langfristig ausgerichteter Psychoedukation (über 1 Jahr) Vorteile sowohl für die psychische Stabilität als auch für das Überleben zeigen.

Die umfangreichste Meta-Analyse wurde von Faller et al. [18] unter Einbeziehung von über 22000 Tumorpatienten aus 198 randomisiert-kontrollierten Studien vorgenommen. Mehr als die Hälfte der Studien kamen aus den USA, eingeschlossen waren überwiegend Patientinnen mit Brustkrebs. In der Analyse wurden signifikante Effekte von Einzel- und Gruppeninterventionen auf emotionalen Distress, Angst, Depression und Lebensqualität nachgewiesen, allerdings bei geringer bis mittlerer Effektstärke. Eine stärkere Wirksamkeit der psychoonkologischen Interventionen konnte für Patienten mit hohem emotionalem Distress bei verlängerter Therapiedauer festgestellt werden, so dass sich unter diesem Aspekt ein Screening der Reha-Patienten auf einen erhöhten Behandlungsbedarf anbietet.

Zur Auswirkung multimodaler Interventionen gibt es nur wenige Studien; auch diese wurden überwiegend bei Brustkrebs durchgeführt. Eine niederländische Arbeitsgruppe fand in einer Übersichtsarbeit für multimodale Konzepte im Vergleich zu «usual care» signifikante Vorteile in Bezug auf Fatigue und körperliche Fitness [19]. Dabei waren multidimensionale Interventionen den Monointerventionen nicht eindeutig überlegen, hierzu konnten allerdings nur wenige Studien ausgewertet werden. Die Kosten-Nutzen-Analyse fiel unter Einbeziehung von 6 Studien überwiegend positiv aus.

\section{Welche Rolle spielt die Ernährung?}

Auf Ernährungsberatung wird in der Rehabilitation traditionell großen Wert gelegt, zumal viele Patienten mit einer Malnutrition (zum Teil therapieinduziert) oder bei fortgeschrittener Erkrankung mit einer Tumorkachexie konfrontiert sind.

Negative Auswirkungen eines raschen Gewichtsverlusts auf die Mortalität sind etwa für Patienten nach allogener Stammzelltransplantation belegt [20].

Es stellt sich darüber hinaus die Frage, ob durch eine Ernährungsumstellung im Sinne einer Tertiärprävention das Rezidivrisiko gesenkt bzw. das Überleben verlängert werden kann. Zu erwarten sind womöglich auch Einflüsse der Ernährung auf Komorbiditäten. In der großangelegten Women's Intervention Nutrition Study (WINS) zeichnet sich nach jetzt 20-jährigem Follow-up ein lebensverlängernder Effekt durch eine fettreduzierte Diät ab [21], allerdings nur bei Frauen mit Hormonrezeptor-negativem frühen Brustkrebs. In anderen Studien wurden diese Ergebnisse teilweise bestätigt - allerdings bei anderen Patientengruppen.

Letztlich ist die Evidenzlage zu Ernährungskonzepten in der Rehabilitation unbefriedigend, da es kaum methodisch gute Studien gibt. Das alte Präventionskonzept mit «täglich fünfmal Obst und Gemüse» ist ebenso wenig auf Sinnhaftigkeit untersucht worden wie der allgemein gehaltene Ratschlag, sich mit gesunder Vollkost $\mathrm{zu}$ ernähren. Eine solche ballaststoffreiche, vitaminhaltige und etwas fettreduzierte Ernährung wird aus empirischen Gründen in der Regel jedoch empfohlen.

\section{Berufliche Interventionen erhalten den Arbeitsplatz}

Die berufliche Wiedereingliederung nach Krebserkrankung ist zweifellos ein großes Problem, denn ein beträchtlicher Anteil der Patienten wird noch immer dauerhaft berentet. In einer MetaAnalyse von 30 Studien, die sich mit körperlichen und psychosozialen Problemen nach der Rückkehr an den Arbeitsplatz befassten, werden die häufigsten Probleme benannt: Demnach haben sich berufstätige Krebspatienten vermehrt mit kognitiven Einschränkungen, mangelnder Krankheitsbewältigung, Depressivität, Angst und hormonellen Störungen (Hitzewallungen, vorzeitige Menopause) auseinanderzusetzen [22].

Hilfe bei der Rückkehr in den Beruf ist zu einem wichtigen Anliegen in der Rehabilitation geworden, zumal sich jeder zweite Krebspatient im erwerbsfähigen Alter befindet. Vor dem Hintergrund eines steigenden Renteneintrittsalters und Fachkräftemangels wird eine auch beruflich orientierte Rehabilitation erwartet, die in Zusammenhang mit den heute besseren Langzeitprognosen der Tumorerkrankungen den Patienten eine Perspektive eröffnet. In diesem Sinne gewinnt die medizinisch-berufliche Orientierung an Bedeutung, obgleich die Studienlage dazu noch schwach ist.

Insgesamt bleibt festzuhalten, dass es bereits viele Studien zur Effektivität nichtmedikamentöser Interventionen in der Rehabilitation gibt, allerdings wird der für Deutschland spezifische Ansatz eines «multimodalen Reha-Settings» darin kaum berücksichtigt. Auch sind die überwiegend untersuchten Einzelinterventionen äußert heterogen, was die Vergleichbarkeit erschwert. Wünschenswert wäre eine offene Kommunikation zwischen den beteiligten Fachärzten und Therapeuten zum Einsatz nichtmedikamentöser Therapiestrategien, die im Zuge einer verbesserten Evidenz auch in Leitlinien berücksichtigt werden sollten. Mit der wachsenden Zahl an Menschen, die Krebserkrankungen langfristig überleben, sind Empfehlungen zur Lebensstilmodifikation wichtiger denn je.

\section{Psychoonkologische Betreuung: Wer gibt welche Hilfe?}

\section{Referentin: Pia Heußner, München}

Eine Krebserkrankung erleben Betroffene und ihre Familien sehr unterschiedlich, weshalb die Frage «Wer braucht welche psychoonkologische Hilfe und in welchem Umfang?» nur schwer zu beantworten ist. Um den Bedarf an Unterstützung abschätzen zu können, sollte man sich zunächst verdeutlichen, wie eine Krebserkrankung durchlebt wird. Es gibt Patienten, die mit großer Kraft und Anpassungsfähigkeit die Erkrankung für sich nehmen können und ihren Weg ohne jegliche Hilfe von außen gehen. Etwa ein 
Drittel der Patienten benötigt jedoch psychoonkologische Unterstützung, entweder weil sie durch die Krebsdiagnose psychisch akut stark belastet sind oder Gefahr laufen, dass sich aus einer solchen akuten Belastung eine langanhaltende Anpassungsstörung oder eine Depression entwickelt.

\section{Das Krankheitserleben ist subjektiv}

Wenn Patienten sich für eine psychoonkologische Begleitung entscheiden, berichten sie zunächst davon, wie sie ihre Krankheit bisher erlebt haben. Sie beschreiben die Krankheitsdiagnose etwa mit dem Bild einer riesigen Flutwelle, von der sie überrollt werden. Ereignisse von solcher Wucht kamen in ihrer bisherigen Biographie nicht vor und übertrafen die Vorstellung eines «schlimmen Ereignisses» bei weitem. Auch von der Angst wird berichtet, in diesem Strudel aus Angst und Verzweiflung unterzugehen. Sie erleben die Krebserkrankung als Super-GAU, als größten anzunehmenden Unfall. Es ist in dieser Phase wenig hilfreich, die Krankheit relativieren $\mathrm{zu}$ wollen, indem auf wirksame Therapien und verbesserte Prognosen hingewiesen wird - subjektiv wird das Geschehen als zerstörerisch und überwältigend erlebt.

\section{Leiden und Heilung haben verschiedene Facetten}

In Anlehnung an die Begründerin der Hospizbewegung, Cicely Saunders, hat Leiden mehr als eine körperliche Dimension. Es findet auch auf einer spirituellen und einer psychosozialen Ebene statt. Dementsprechend besteht auch die spätere Gesundung nicht nur aus der Wiederherstellung der körperlichen Funktionsfähigkeit, sondern hat auch seelische Facetten.

Auch wenn aus schulmedizinischer Sicht unter Einbeziehung sämtlicher Informationsquellen bis hin zum Genotyping die Prognose einer individuellen Krebserkrankung als gut einzuschätzen ist, wird diese Information vom Patienten unter Umständen anders als zu erwarten verarbeitet. Abgesehen davon, dass prognostische Aussagen stets die Unsicherheit einer wissenschaftlichen Abschätzung beinhalten, besteht auch eine Diskrepanz zwischen dem, was der Arzt sagt und wie der Patient den weiteren Krankheitsverlauf selbst erwartet. Krebs wird trotz aller medizinischen Fortschritte in der Gesellschaft oft mit Leiden und Tod in Verbindung gebracht, so dass die Denkweise «diese Krankheit wird mich früher oder später umbringen» fest im Kopf verankert sein kann. Eine günstige Prognose allein, die womöglich in Aussicht gestellt werden kann, wirkt auf den Patienten nicht entlastend. Er braucht in dieser ihn verunsichernden Situation zunächst eine Art Rettungsring, eine Handreichung, das Aufzeigen eines für ihn bislang nicht erkennbaren Wegs. Es geht darum, zunächst Zutrauen in die vorgeschlagene Therapie zu vermitteln. Dabei ist zu akzeptieren, dass es auch Patienten gibt, denen es nicht um eine maximale Verlängerung der Lebenszeit geht, sondern die mögliche Therapieerfolge gegen den Verlust an Lebensqualität abwägen.

\section{Phasen der Krankheitsbewältigung}

Ebenso wie das Leiden definiert sich die persönliche Lebensqualität nicht nur über körperliche Aspekte, sondern auch über spirituelle und psychosoziale. Jeder Patient entscheidet für sich, welche

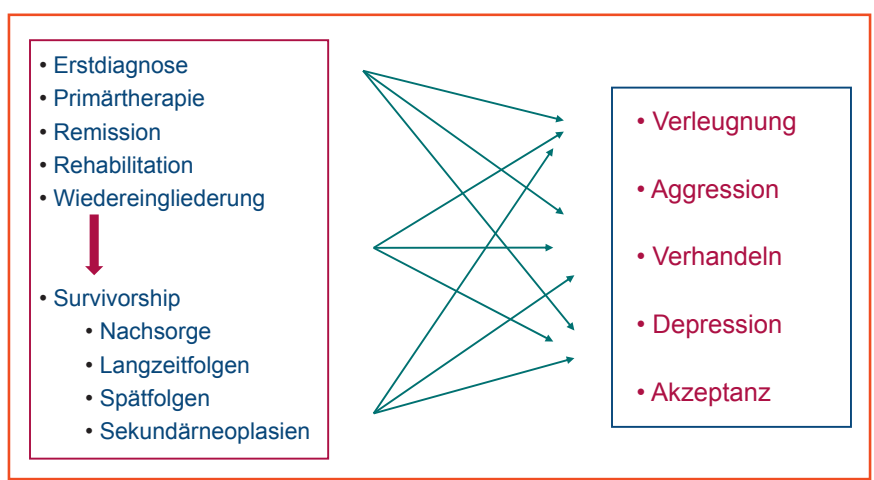

Abb. 6. Psychische Reaktionen und Krankheitsverarbeitung im Krankheitsverlauf.

Erwartungen er an seine verbleibende Lebenszeit hat und wie wichtig ihm Lebensinhalte und Lebensfreude sind. Diese Präferenzen können sich im Verlauf einer Erkrankung ändern; das gilt auch für die Auffassung darüber, wieviel Verlust an Lebensqualität hinnehmbar ist.

Im Verlauf einer Krebserkrankung wird der Patient immer wieder mit psychischen Belastungen konfrontiert. Das beginnt mit dem Auftauchen der ersten Symptome und gilt für die Phasen der Therapie, Remission und Rehabilitation bis hin zur Nachsorge sowie unter Umständen für die schwierige Situation bei weiterer Progredienz. Die klassischen Phasen der Krankheitsverarbeitung von der Verleugnung, über Aggression, Verhandeln, Depression und Akzeptanz verlaufen nicht linear, was die Zuordnung zu den verschiedenen psychologischen Hilfsangeboten vereinfachen würde. Vielmehr können die verschiedenen Schritte der Krankheitsverarbeitung im Verlauf der Erkrankung mehrfach durchlaufen werden, es kann zwischenzeitlich eine Stagnation geben, eine retrograde oder eine sprunghafte Weiterentwicklung. Weil die Zusammenhänge zwischen Krankheitsverlauf und Krankheitsbewältigung so komplex sind, müssen auch die Betreuungsangebote individuell und flexibel aufgebaut sein.

Patienten, die aller Voraussicht nach ihre Krankheit lange überleben werden, stehen vor der Aufgabe der Wiedereingliederung in das Leben nach Abschluss der Reha-Phase (Abb. 6). Dies erfordert das Finden einer neuen Normalität in Alltags- und Freizeitaktivitäten sowie die berufliche und soziale Wiedereingliederung. Die Aufgabe besteht darin, den Platz im Leben nach alter Gewohnheit wieder einzunehmen oder das Verständnis vom erfüllten Leben neu zu definieren. Dabei bleibt auch die überstandene Krebserkrankung weiterhin präsent - sei es bei den Nachsorgeterminen, die von vielen Patienten als stets belastend erlebt werden, oder im Umgang mit chronischen Krankheitszeichen, Langzeit- und Spätfolgen der Krebstherapie oder Sekundärtumoren.

Patienten setzen sich sehr unterschiedlich mit den Besonderheiten des Survivorship auseinander, etwa in Bezug auf die Nachsorge. Diese in regelmäßigen Abständen angesetzten Kontrollen sind oft angstbesetzt. Insbesondere die «Wartezeit» zwischen der Untersuchung und der Befundmitteilung wird als äußerst belastend erlebt. Es hilft den Patienten sehr, diese Zeit der Unsicherheit so kurz wie 
eben möglich zu halten. Es gibt aber auch Patienten, denen die Zeit zwischen zwei Nachsorgeterminen zu lang erscheint. Sie brauchen die Bestätigung, dass alles in Ordnung ist, in kurzen Intervallen. Der Angst und Unsicherheit wird von diesen Patienten gelegentlich dadurch begegnet, dass sie die Abstände zwischen den Nachsorgeterminen verkürzen, indem sie mehrere Ärzte mit Kontrolluntersuchungen beauftragen.

Viele Langzeitüberlebende sehen sich auch mit Langzeitkomplikationen und Spätfolgen ihrer Therapie konfrontiert. Besonders die Spätfolgen, etwa kardiale Schädigungen nach Thoraxbestrahlung oder Antrazyklin-haltiger Chemotherapie, treffen die Patienten mitunter völlig überraschend. Da oft schon viele Jahre seit der Krebserkrankung vergangen sind, sind die Zusammenhänge nur noch schwer nachzuvollziehen. Ebenso schwierig ist es für die Patienten, mögliche Spätfolgen schon zur Therapieentscheidung abzuwägen. Dies betrifft z.B. die Auseinandersetzung mit einer möglichen Infertilität nach bestimmten Krebstherapien. Besonders junge Patienten, für die das Thema Kinderwunsch vor ihrer Erkrankung noch keine Rolle spielte, können sich bedrängt fühlen. In dieser Situation mögen dann Entscheidungen getroffen werden, die von den Patienten später in Frage gestellt werden. Im Rahmen einer psychoonkologischen Beratung können Ängste und Abwehrhaltungen zu diesem Thema reflektiert und teilweise bearbeitet werden.

\section{Risiko von Zweitkarzinomen wird ignoriert}

Wird bei Patienten nach einer Tumorerkrankung später erneut Krebs diagnostiziert - etwa eine sekundäre Leukämie nach Therapie eines Mammakarzinoms -, so wird das als besonders traumatisch erlebt. Die Betroffenen haben im Rahmen ihrer Einwilligung zur Therapie des Primärkarzinoms zwar vom erhöhten Risiko für Zweitmalignome gehört, sich damit aber in der Regel nicht mental auseinandergesetzt. Patienten müssen bekanntlich besonders zu Beginn einer Krebstherapie so viele Entscheidungen treffen, dass in ferner Zukunft liegende Risiken nicht prioritär bedacht werden können. Kommt es dann zur Entwicklung eines weiteren Malignoms, können alle Phasen der Krankheitsverarbeitung erneut durchlaufen werden.

\section{Ziele der Rehabilitation für Langzeitüberlebende}

Für Langzeitüberlebende, die sich mit den geschilderten Problemen des Survivorship auseinandersetzen müssen, ist eine konventionelle dreiwöchige stationäre Rehabilitation nur ein Tropfen auf den heißen Stein. Die für gewöhnlich angestrebten Reha-Ziele sind eine Verbesserung der physischen Kondition, Abbau psychischer Belastungen, Einfinden in eine neue Normalität sowie eine Neuorientierung im sozialen Umfeld in Bezug auf Familie, Freunde, Beruf, Hobby und vieles mehr. Diese Ziele lassen sich nicht kurzfristig umsetzen. Es mag ein halbes Jahr dauern, bis eine Neuorientierung gelingt, vielleicht auch länger. $\mathrm{Zu}$ unterschiedlich sind die Anpassungsleistungen, die Langzeitüberlebende entsprechend ihrer persönlichen Lebenssituation erbringen müssen. Manche müssen sich über viele Jahre mit Belastungen arrangieren, die von ihrer lang zurückliegenden Krebserkrankung herrühren. Nicht immer reagiert das Umfeld darauf mit Verständnis. Es wird erwar-
- Unverständnis

- Enttäuschung

- Resignation

- emotionale

Erschöpfung

- Isolation

- Depression

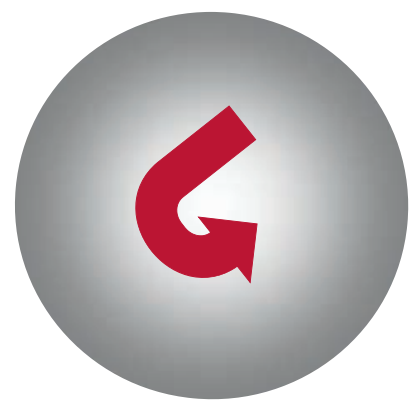

Abb. 7. Folgen der misslingenden psychischen Rehabilitation.

tet, dass eine Krebserkrankung spätestens nach mehrfachen RehaAufenthalten psychosozial «verarbeitet» worden ist und der ehemalige Krebspatient wieder rundum «gesund» ist. Gelingt dies im Rahmen der Rehabilitation nicht, können Patienten resignieren und chronisch psychisch krank werden (Abb. 7). Dies betrifft zwar nur wenige Patienten, hat aber für die davon Betroffenen und ihre Familien erhebliche Auswirkungen.

\section{Wer kann den Patienten helfen?}

Die psychoonkologische Betreuung der Langzeitüberlebenden liegt in erster Linie bei ihren Onkologen und Hausärzten. Sie sind oft bereits seit Jahren die erste Anlaufstelle, an die sich Patienten mit ihren Fragen und Sorgen wenden. Onkologen und Hausärzte können die Lebensumstände der Patienten am ehesten überblicken und frühzeitig erkennen, ob es ungelöste Konflikte im Zusammenhang mit der Krebserkrankung gibt. Alle anderen Beratungsstellen wissen weit weniger über den Krankheitsverlauf des Patienten, die medizinischen Hintergründe der Erkrankung und den bisherigen Umgang des Patienten mit seiner Erkrankung. Die Psychoonkologischen Dienste (POD) der Akutkliniken, die Psychosozialen Krebsberatungsstellen, die Reha-Einrichtungen, Selbsthilfegruppen und niedergelassenen psychoonkologisch orientierten Psychotherapeuten sind letztlich auf Hilfestellung im psychosozialen Bereich spezialisiert. Für die meisten Patienten ist es aber sinnvoll, einen einzigen Ansprechpartner zu haben, der die medizinischen Sachverhalte kennt und auch für psychosoziale Probleme ein offenes Ohr hat.

Auch wenn in den Praxen der niedergelassenen Kollegen Zeitund Kostendruck herrschen, wird dieser ganzheitliche Anspruch oft mit viel Engagement im Rahmen der Möglichkeiten umgesetzt. Für manche Fragestellungen und höhergradige psychische Belastungen ist es notwendig, spezialisierte Hilfe hinzuzuziehen. Es gibt Einrichtungen, die ohnehin eng mit psychoonkologischen Beratungen kooperieren und diese Betreuungsaufgabe gewissermaßen auslagern. Krebszentren schließlich müssen einen POD im Rahmen der Zertifizierungsauflagen vorhalten. Dabei ist zu hinterfragen, wie diese Dienste personell besetzt sind und über welche Kompetenz die Mitarbeiter verfügen. Nicht zu unterschätzen ist die Arbeit der kommunalen Psychosozialen Krebsberatungsstellen, die insbesondere auch Aufgaben der Sozialdienste übernehmen und z.B. zu Fragen in Sachen Rente oder Schwerbehinderung beraten. Diese 
Abb. 8. 5-Jahres-Überleben in Abhängigkeit vom sozioökonomischen Status (modifiziert nach [27]).

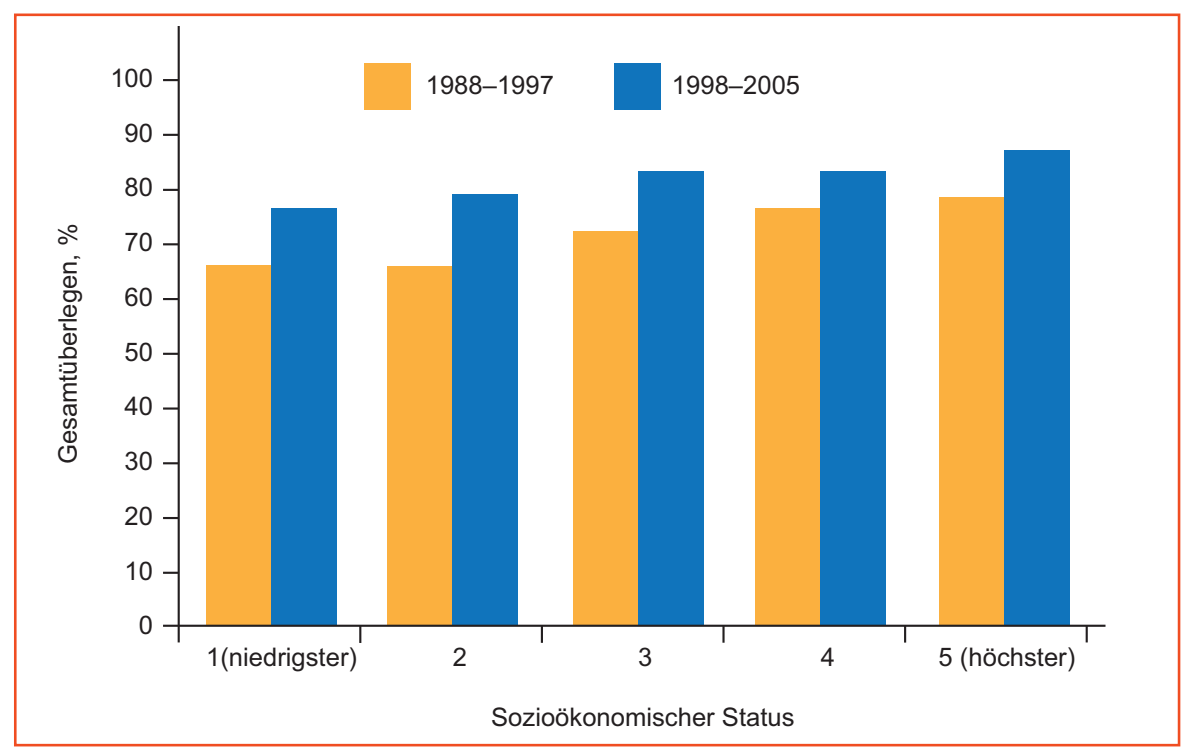

Krebsberatungsstellen unterliegen nicht der Regelfinanzierung der Krankenkassen, sondern werden über Fördermittel der Deutschen Krebshilfe finanziert oder sind in karitativer Trägerschaft.

Auf die Möglichkeiten der psychoonkologischen Intervention in stationären Reha-Einrichtungen wird an anderer Stelle eingegangen. Im Anschluss an die Rehabilitation schließen sich manche Patienten einer Selbsthilfegruppe an. Diese können eine gute Hilfestellung sein, besonders dann, wenn die Gruppen gut moderiert werden. Es ist dann durchaus möglich, dass auch ungünstige Krankheitsverläufe einzelner Gruppenmitglieder von allen mitgetragen und miterlebt werden, ohne dass die eigene Krankheitsbewältigung darunter leidet.

Für Patienten mit ausgeprägten psychischen Belastungen, die auf ihrem Weg in ein neues Leben Unterstützung brauchen, stehen psychoonkologisch spezialisierte Psychotherapeuten zur Verfügung. Sie können Patienten mit einer Angststörung oder Depression im Rahmen der kassenärztlichen Versorgung behandeln. Fehlt eine im Leistungskatalog der Kassen abgebildete Diagnose, ist die Finanzierung einer psychoonkologischen Begleitung nicht gesichert. In jedem Fall ist ein psychotherapeutisches Gutachten nötig, wenn eine Kostenübernahme seitens der Kassen angestrebt wird. Dieser Aufwand wird häufig gescheut, weshalb die psychoonkologische Betreuung außerhalb onkologischer Zentren lückenhaft ist.

\section{Identifikation von Risikopatienten}

\section{Referent: Ulf Seifart, Marburg, für den Arbeitskreis onkologische Rehabilitation in der DGHO}

Die Zahl der Langzeit-Survivors von Krebserkrankungen wird weiter zunehmen, da sich bei steigender Krebsinzidenz die Prognose der Patienten stetig verbessert hat [23]. Von den in Deutschland aktuell etwa 1,4 Millionen Menschen mit Krebserkrankungen sind etwas mehr als die Hälfte im erwerbsfähigen Alter. Laut Da- tenerhebung kehrt von ihnen wiederum nur etwa die Hälfte nach der Krebstherapie in das Berufsleben zurück [24]. Damit stehen jedes Jahr geschätzte 100000 Tumorpatienten vor der Frage, ob und wie die Rückkehr in den Beruf gelingen kann. Dies ist auch ein gesellschaftliches Problem, denn die Kosten wegen Arbeitsunfähigkeit infolge einer Tumorerkrankung belaufen sich europaweit auf fast 10 Milliarden EUR [25].

Nicht arbeiten zu können bedeutet für die Betroffenen aber auch eine Einschränkung ihrer Lebensqualität. Gemäß einer Umfrage unter 149 Patienten mit fortgeschrittenen Tumoren waren Finanzsorgen unabhängig vom Einkommen eine signifikant größere Belastung als mögliche körperliche oder psychische Beeinträchtigungen durch die Erkrankung [26]. Dies gilt sicher nicht für alle Patienten, weist jedoch darauf hin, dass die ökonomischen Folgen einer Tumorerkrankung ein relevantes Problem sein können. In einer US-amerikanischen Studie korrelierte ein niedriger sozioökonomischer Status mit einem schlechteren 5-Jahres-Gesamtüberleben [27]. Dies galt sowohl für die 1990er Jahre als auch für den Zeitraum bis 2005 (Abb. 8).

In einer Subgruppe von 2835 Teilnehmerinnen der Nurses' Health Study, die im Verlauf dieser Längsschnittstudie an Brustkrebs erkrankt waren, hatten sozial schlecht integrierte Frauen eine um $66 \%$ erhöhte Gesamtmortalität und eine zweifach erhöhte Brustkrebsmortalität [28]. Auch in Untersuchungen aus Schweden bei Patienten mit hämatologischen Malignomen zeigte sich, dass Patienten in gehobenen beruflichen Positionen («white collar patients») eine signifikant geringere Mortalität hatten als Patienten aus anderen sozialen Gruppen [29, 30]. Erst kürzlich wurde eine Meta-Analyse US-amerikanischer Daten publiziert, wonach an Leukämie erkrankte Kinder aus einkommensschwachen Familien eine ungünstigere Prognose haben als Kinder aus wohlhabenderen Familien [31]. Für Deutschland zeigte eine Auswertung von zehn Krebsregistern, die insgesamt 200 Einzugsbereiche abdeckten, dass Tumorkranke aus einkommensstarken Regionen bessere 5-Jahres-Überlebensraten erreichten als Krebspatienten aus sozial schwächeren Gegenden [32]. 


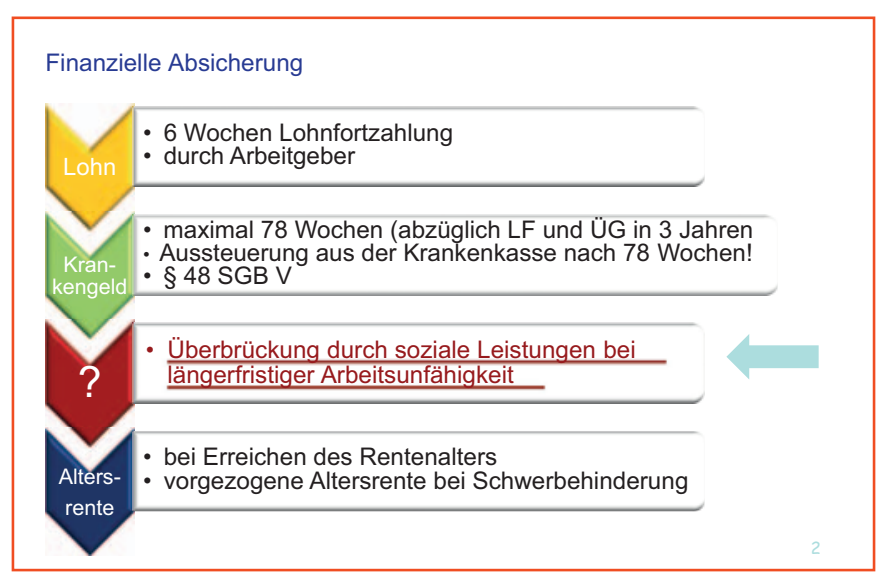

Abb. 9. Arbeitsunfähigkeit und soziale Sicherungssysteme.

\section{Arbeitsunfähigkeit führt zu finanzieller Not}

Die aus der verminderten Leistungsfähigkeit resultierende $\mathrm{Ar}$ beitsunfähigkeit kann für die Betroffenen zu einer großen finanziellen Belastung werden, die allein durch die sozialen Sicherungssysteme wie Lohnfortzahlung und Krankengeld häufig nicht aufgefangen werden kann (Abb. 9).

Eine Zäsur bedeutet insbesondere die Aussteuerung aus der Krankenkasse nach etwa 1,5 Jahren anhaltender Arbeitsunfähigkeit. Zu diesem Zeitpunkt wird die Zahlung des Krankengelds in der Regel ohne entsprechende Ankündigung eingestellt. Trifft dies den Patienten unvorbereitet, muss die sich auftuende finanzielle Lücke kurzfristig durch andere soziale Leistungen überbrückt werden, was aufgrund längerer Bearbeitungszeiten bei der Antragsprüfung zum Problem werden kann. Wer nicht direkt Altersrente bezieht, läuft Gefahr mit der Aussteuerung aus der Krankenkasse in die Arbeitslosigkeit zu geraten

Eine Meta-Analyse von 36 Studien zeigte, dass Langzeit-Krebsüberlebende häufiger arbeitslos sind als gleichaltrige Kontrollpersonen $[33,34]$. Dies war auch das Ergebnis einer systematischen Literaturdurchsicht von insgesamt 64 Studien [24]. Demnach kehrten etwa 64\% der Survivors in das Erwerbsleben zurück. Die mittlere Zeit der Arbeitsunfähigkeit betrug 151 Tage. Bis zur Hälfte der Patienten verlor zunächst ihren Arbeitsplatz. Die Mehrzahl von ihnen fand später einen neuen Arbeitsplatz, allerdings häufig in Form einer Teilzeittätigkeit. Auch aus den Daten der DRV zur Erwerbstätigkeit von Patienten nach onkologischer Rehabilitation geht hervor, dass lediglich die Hälfte von ihnen zum Zeitpunkt der Rehabilitation laufende Beitragszahler sind.

Patienten, die nach Bezug von Lohnfortzahlung und Krankengeld arbeitslos werden, sind Bezieher von Arbeitslosengeld 1, das derzeit für Durchschnittsverdiener 840 EUR/Monat beträgt. Dies ist mit einem erhöhten Risiko für Verarmung und soziale Isolation verbunden [35]. Wer alternativ eine Erwerbsminderungsrente bezieht, ist eher noch schlechter gestellt und häufig auf zusätzliche Leistungen wie Wohngeld angewiesen, wenn der «Haupt Ernährer» der Familie betroffen ist. Welche Patienten gefährdet sind, in eine solche negative Spirale zu gelangen, konnte im Rahmen einer nichtrepräsentativen, von den hessischen Krebsberatungsstellen

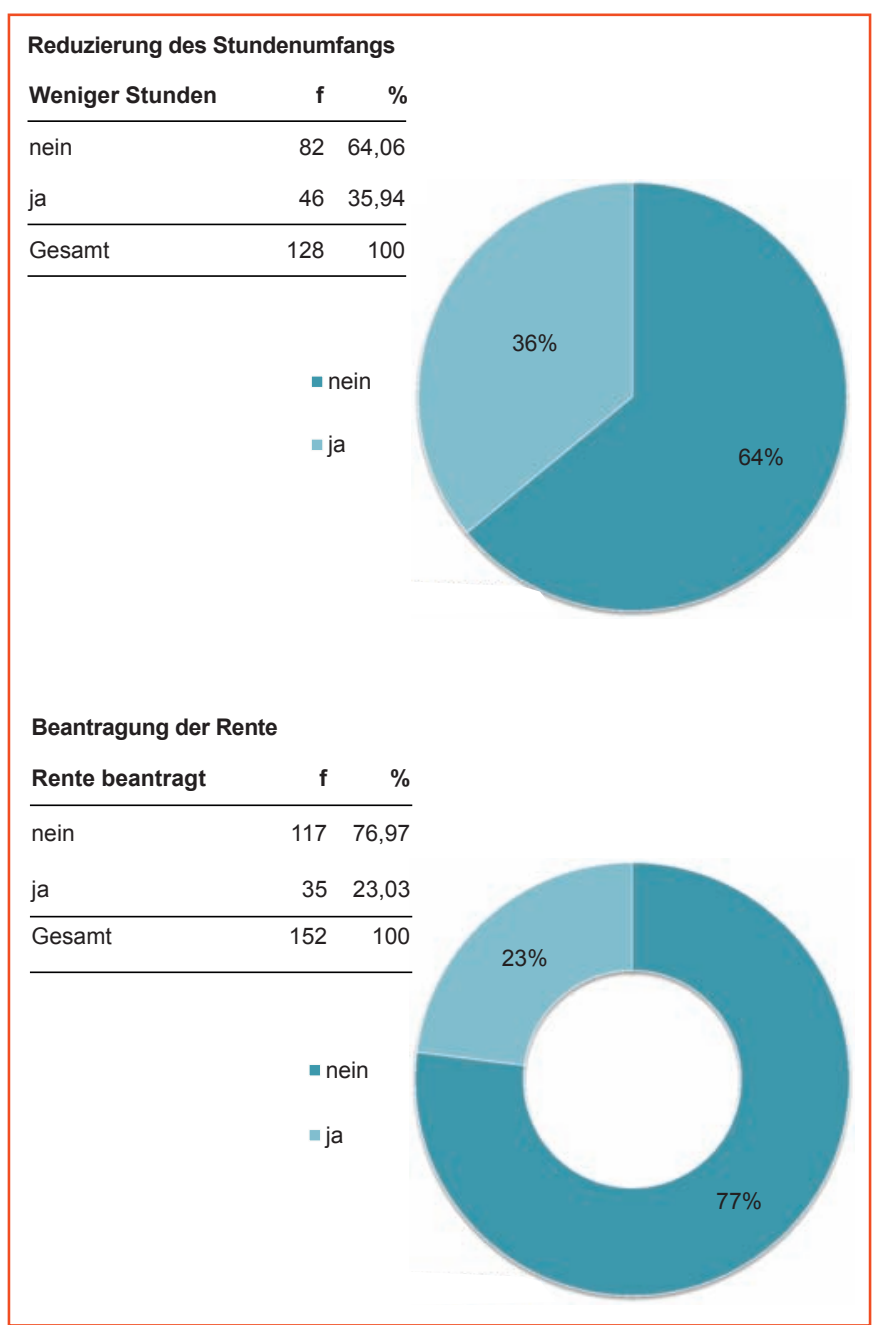

Abb. 10. Krebserkrankung und die Folgen für die Berufstätigkeit.

vorgenommenen Befragung von 198 Patienten bzw. deren Angehörigen eingegrenzt werden. Es handelte sich zu 80\% um an Brustkrebs erkrankte Frauen. Ein jeweils größerer Teil der Befragten hatte zum Zeitpunkt der Datenerhebung (Juni bis Oktober 2014) den Umfang der Berufstätigkeit reduziert und/oder einen Antrag auf Rentenbezug gestellt. (Abb. 10). Dies betraf überproportional häufig Patienten mit niedriger Bildung und schwerer körperlicher Arbeit, wobei die geringen Gruppengrößen keine belastbare statistische Auswertung erlauben.

Eine weitere kleine Umfrage unter insgesamt 76 unserer allogen transplantierten Patienten mit einer hämato-onkologischen Erkrankung hat ergeben, dass immerhin $37 \%$ von ihnen 1 Jahr nach der Transplantation wieder berufstätig waren, davon allerdings die Hälfte mit Einschränkungen. 26\% der Befragten bezogen eine Zeitrente, $25 \%$ Krankengeld und $1 \%$ eine Altersrente. 3\% der Umfrageteilnehmer war arbeitslos. Alle Patienten, die wieder beruflich eingegliedert werden konnten, übten ihre Tätigkeit vom Schreibtisch aus.

Als Risikofaktoren für eine anhaltende Nichterwerbsfähigkeit erwies sich auf Grundlage dieses kleinen Daten-Pools ein Lebensalter von über 40 Jahren und eine körperlich mittelschwere bis 
Ungünstige Faktoren

- Chemotherapie

- Rezidiv

- ein fortgeschrittenes Tumorstadium

- und zusätzlich andere chronische Erkrankungen prognostisch ungünstige Faktoren

- Im Rahmen einer COX-Regressionsanalyse fanden Spelten et al. [35] anhand von 235 Tumorpatienten die Angabe von körperlichen Beschwerden als auch das Fatigue Syndrom als wesentliche Ursache für eine Arbeitsunfähigkeit.

Abb. 11. Ungünstige Prädiktoren für künftige Berufstätigkeit

schwere Arbeit. Patienten mit solcher Konstellation sollte dringend zu einer onkologischen Rehabilitation geraten werden, da im Rahmen einer solchen Maßnahme auch die beruflichen Perspektiven ausgelotet werden können. Im Übrigen stand die Arbeitsunfähigkeit nicht in Zusammenhang mit der Krankheitsdiagnose, der Schulbildung, möglichen Komplikationen der Transplantation und dem HLA-Status.

Auch in dem bereits zitierten Literatur-Review wurde der Frage nachgegangen, ob es Prognosefaktoren für die weitere Erwerbsfähigkeit von Patienten mit Tumorerkrankungen gibt [36]. In Bezug auf die Tumorentität waren Leber- und Lungenkrebs, fortgeschrittene Blut- und Lymphdrüsenkrebserkrankungen, Hirntumoren, Pankreaskarzinome sowie Kopf-Hals-Tumoren mit einem deutlich höheren Risiko für Langzeitarbeitslosigkeit verknüpft. Dagegen hatten Patienten mit Tumoren des Urogenitaltrakts, HodgkinLymphomen und Brustkrebs bessere Aussichten auf eine erfolgreiche berufliche Wiedereingliederung. Keine statistisch signifikanten Unterschiede fanden sich bei Patienten mit Hodentumoren und malignen Melanomen im Vergleich zu Nicht-Tumorpatienten. Das Risiko für Arbeitslosigkeit stieg darüber hinaus mit der Intensität der Chemotherapie und weiteren tumorassoziierten Faktoren (Abb. 11). In einer früheren Studie unter Einbeziehung von 235 Krebspatienten war bereits das Fatigue-Syndrom als weitere wesentliche Ursache für eine Arbeitsunfähigkeit identifiziert worden [36].

In der von Mehnert [24] vorgenommenen Meta-Analyse fanden sich aber auch eine Reihe von Faktoren, die sich prognostisch günstig auf die berufliche Wiedereingliederung auswirkten (Abb. 12).

Der aus den Studien eruierte günstige Prognosefaktor der professionellen Hilfe bei der Wiederaufnahme der beruflichen Tätigkeit lässt sich am besten im Rahmen einer Rehabilitation generieren. Das Hinarbeiten auf den Erhalt des Arbeitsplatzes gehört zu den essentiellen Aufgaben der Rehabilitation. Die Möglichkeiten, auf den Erhalt der Arbeitsfähigkeit hinzuwirken, sind in Rahmen einer Rehabilitation deutlich größer als im Gespräch mit dem Fach- oder Hausarzt. In einer Auswertung von über 1000 onkologischen Patienten, die vor Beginn der Rehabilitation erwerbsunfähig waren, zeigte sich der Erfolg der Reha-Maßnahme in Bezug auf die berufliche Zukunft: 97\% der Angestellten konnten nach erfolg-
Günstige Prognosefaktoren

- unmittelbaren Verhältnisse am Arbeitsplatz und die Hilfestellung bei der Rückführung ins Erwerbsleben.

- gute Einbindung in das soziale Gefüge am Arbeitsplatz

- durch den Arbeitgeber zuträgliche Arbeitsbedingungen geschaffen werden

- professionelle Hilfe bei der Wiederaufnahme der beruflichen Tätigkeit besteht

- ist die Wahrscheinlichkeit für eine dauerhafte Erwerbsfähigkeit höher als wenn diese Faktoren nicht oder soaar aeaenteilia vorhanden sind.

Abb. 12. Günstige Prädiktoren für künftige Berufstätigkeit.

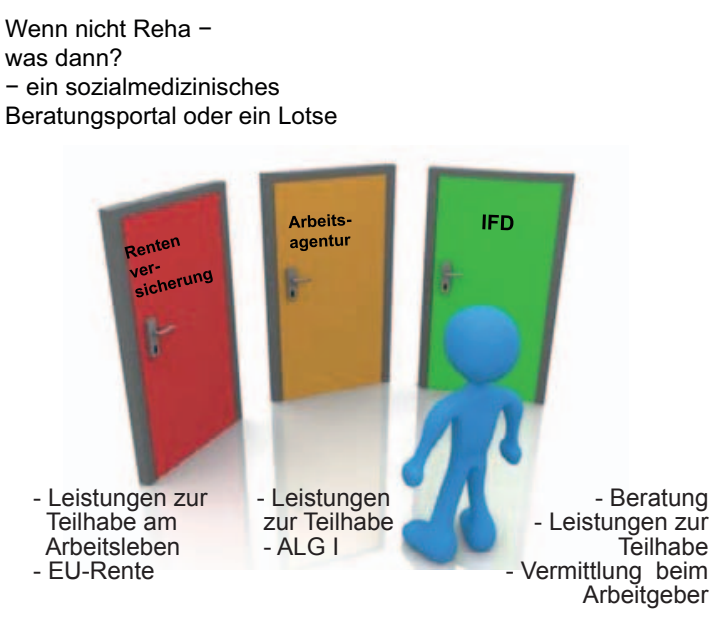

Abb. 13. Das sozialmedizinische Angebot ist umfangreich und schwer zu überblicken.

reichem Abschluss der Rehabilitation wieder eine Berufstätigkeit aufnehmen [37]. Bei den Arbeitern gelang dies allerdings nur zu $56 \%$, was die Notwendigkeit unterstreicht, insbesondere für diese Gruppe der Berufstätigkeiten das Reha-Angebot zu verbessern. Wünschenswert sind auch ein sozialmedizinisches Beratungsportal und ein Lotse, der bei der beruflichen Wiedereingliederung oder der Beantragung von Sozialleistungen hilft. Das Leistungsanbot wird von verschiedenen Institutionen wie der DRV oder dem Integrationsfachdienst vorgehalten und ist vom Patienten und auch von den betreuenden Fach- und Hausärzten nur schwer zu überblicken (Abb. 13). Von daher benötigen die Patienten in diesen Fragen professionelle Hilfe

Der Sozialdienst der Akutkliniken ist in dieser Thematik häufig nicht mehr zuständig, da die Patienten entlassen bzw. ambulant betreut werden. Fernerhin bestehen aufgrund der Stellenreduktionen in diesem Bereich häufig personelle Engpässe. Die Beratungsstellen der Krebsgesellschaften bieten in diesen Aufgaben eine suffiziente Betreuung an, allerdings steht zu befürchten, dass die Finanzierung dieser Stellen über das Jahr 2016 hinaus gefährdet ist. Hier ist zu hoffen, dass eine Regelung im Sinne der Patienten gefunden wird. 
Nachsorge und Prophylaxe von Langzeitkomplikationen - was ist evidenzbasiert?

\section{Referentin: Georgia Schilling, Freiburg i.Br.}

Multimodale Therapiekonzepte in der Onkologie, die lokale Verfahren wie Operation und Strahlentherapie mit systemischen Behandlungen kombinieren, haben häufig einen kurativen Ansatz. Unter Nutzung solch moderner Therapiestrategien hat sich die Prognose vieler Patienten in Bezug auf ihr Langzeitüberleben deutlich gebessert. Eine Reihe neuerer Krebsmedikamente kann dauerhaft eingenommen bzw. infundiert werden, um einen Tumorprogress oder eine Fernmetastasierung zu vermeiden, so dass aus dem akuten Tumorgeschehen eine chronische Krebserkrankung wird, die mit einer Dauer- oder Erhaltungstherapie effektiv kontrolliert werden kann. Die komplexen, interdisziplinären Therapiestrategien verlängern jedoch nicht nur das Überleben und vergrößern die Heilungschancen, sondern potenzieren auch Spätkomplikationen und unerwünschte Langzeitwirkungen.

It's not over when it's over...

Solche unerwünschten Langzeiteffekte können während oder nach Abschluss einer Therapie auftreten und sind eine Belastung für Patienten, die als Krebs-Survivor in ein neues Leben starten wollen. $\mathrm{Zu}$ den zahlreichen möglichen Begleiteffekten einer Krebstherapie gehören pulmonale Toxizität, Kardiotoxizität, Polyneuropathien, «Chemobrain», Fatigue, Zweitmalignome, Infertilität und Schmerzen.

\section{Das Problem der kardialen Spättoxizität}

Die relativ hohe Kardiotoxizität bestimmter Krebsmedikamente z.B. aus der Gruppe der Anthrazykline kann den langfristigen Behandlungserfolg limitieren. So kann etwa eine dilatative Kardiomyopathie nach adjuvanter Doxorubicin-Gabe die Lebensqualität der Patienten erheblich beeinträchtigen. In einer Studie an über 1500 Patienten mit Hodgkin-Lymphomen konnte gezeigt werden, dass allein die mediastinale Bestrahlung das Risiko für kardiovaskuläre Erkrankungen um das Zwei- bis Siebenfache erhöhte [38]. Unter Anthrazyklin-haltigen Chemotherapien stieg das Risiko für ein kongestives Herzversagen und vaskuläre Erkrankungen um den Faktor 2-3. Das Risiko nahm über die Jahre stetig zu und betrug in Bezug auf die kardiovaskuläre Ereignisrate 25 Jahre nach Radiochemotherapie fast $8 \%$. Die Patienten hatten außerdem über Jahrzehnte ein erhöhtes Risiko für eine klinisch relevante kardiale Dysfunktion.

Unter den Anthrazyklinen hat besonders Doxorubicin eine erhebliche Kardiotoxizität $[39,40]$. So entwickelte sich nach adjuvanter Therapie mit Doxorubicin bei Gabe kumulativer Höchstdosen von $500-600 \mathrm{mg} / \mathrm{m}^{2}$ in $4-36 \%$ der Patienten eine objektivierbare Herzinsuffizienz. Auch bei Verwendung einer niedrigen Dosis (240-300 mg/m $\left.\mathrm{m}^{2}\right) \mathrm{kam}$ es bei immerhin 1\% der Patienten zu einer Herzschädigung, weshalb neuere Therapieleitlinien bereits ab einer kumulativen Doxorubicin-Dosis von $300 \mathrm{mg} / \mathrm{m}^{2}$ ein engmaschiges kontinuierliches Monitoring empfehlen.
Die Symptomatik der strahlentherapieassoziierten Herzerkrankungen (radiation-induced heart diseases; RHID) reicht von klinisch inapparenten histopathologischen Veränderungen bis hin zur manifesten Erkrankung bei ca. 10\% der Patienten [41]. Risikofaktoren für eine symptomatische RHID umfassen eine vorangegangene Chemotherapie, Strahlendosen $>30$ Gy und eine linksthorakale Bestrahlung. Für Patienten, die in der Kindheit bestrahlt wurden, ist das Risiko der kardialen und kardiovaskulären Mortalität selbst bei relativ geringen Strahlendosen erheblich erhöht. Die kumulative Inzidenz kardialer Ereignisse nach Bestrahlung und insbesondere nach Anthrazyklin-haltiger Radiochemotherapie nimmt mit zeitlichem Abstand zur Therapie kontinuierlich zu [42].

\section{Kardiales Monitoring erforderlich}

Auch die Therapie mit zielgerichteten Substanzen ist mit einem erhöhten kardialen Risiko verbunden. Dies gilt unter anderem für den Antikörper Trastuzumab, unter dem in Studien vermehrt schwere Herzinsuffizienzen (1\%), objektivierbare kardiale Dysfunktionen (5\%) und symptomatische Herzinsuffizienzen (2\%) auftraten. Auch bei Lapatinib ist von einer erhöhten Kardiotoxizität auszugehen, jedoch nicht bei den neueren Anti-HER2-gerichteten Substanzen Pertuzumab und Trastuzumab-Emtansin .

Im Gegensatz zu den Anthrazyklinen ist die Trastuzumab-induzierte Kardiomyopathie nicht streng dosisabhängig und in der Regel vollständig reversibel. Außerdem steigt das Risiko mit den Jahren nicht weiter an [43]. Mittlerweile wird von Fachgesellschaften zwischen den verschiedenen Formen der therapieassoziierten Kardiotoxizität (Anthrazykline vs. andere Therapien) unterschieden [44]. Ferner gibt es Empfehlungen zum Monitoring der Kardiomyopathien [45], das sich hauptsächlich auf die Echokardiographie und die Bestimmung des Serummarkers Troponin stützt.

Zur Minderung chronischer Kardiotoxizität bei Patientinnen mit fortgeschrittenem Mammakarzinom, die bereits eine kumulative Anthrazyklin-Dosis von über $300 \mathrm{mg} / \mathrm{m}^{2}$ Doxorubicin oder $540 \mathrm{mg} / \mathrm{m}^{2}$ Epirubicin erhalten haben und deren Therapie mit Anthrazyklinen fortgesetzt werden soll, steht Dexrazoxan zur Verfügung [46, 47]. Der intrazelluläre Eisenchelat-Bildner ist seit 2007 ausschließlich innerhalb dieses engen Indikationsbereichs zugelassen und darf weder bei anderen Tumorentitäten noch bei Patienten mit initialer Adriamycin-Therapie eingesetzt werden.

Für Krebspatienten, die bereits eine Kardiomyopathie entwickelt haben, stehen die üblichen Therapieoptionen bei Herzinsuffizienz zur Verfügung (Abb. 14).

Grundsätzlich ist es sinnvoll, eine Herzinsuffizienztherapie frühzeitig zu beginnen, um einen weiteren Abfall der linksventrikulären Ejektionsfraktion (LVEF) zu verhindern. Im Rahmen einer Studie sprachen Patienten mit einer LVEF < 45\% umso besser auf eine Kombinationstherapie mit Enalapril plus Carvedilol an, je früher damit begonnen wurde [49]. Therapie-Responder hatten zudem weniger kardiale Ereignisse. In einer weiteren kontrollierten Studie wurde die Wirksamkeit von Telmisartan bei Epirubicininduziertem frühem Myokardschaden untersucht [50]. Mit dem Sartan konnte im Vergleich zu Placebo die LVEF signifikant besser 
- ACE-Hemmer (AT-II-Rezeptorblocker)

- $\beta$-Blocker

- Ziel: RR <140/90 mm Hg; Hf 70/min

- Aldosteron-Antagonisten

- Digitalis

- Diuretika

- allgemeine Maßnahmen: Flüssigkeitsrestriktion (1,5 I), körperliche Bewegung, gesunde Ernährung, salzarme Kost

Abb. 14. Therapie bei Herzinsuffizienz als Spätkomplikation einer Krebstherapie (modifiziert nach [48]).

stabilisiert werden und erreichte nach einem Follow-up von 12 Monaten wieder den Normbereich.

Eine Vorhersage der Kardiotoxizität kann offenbar unter Heranziehung genetischer Polymorphismen (SNPs) in Kombination mit bekannten Risikofaktoren (kumulative Gesamtdosis des Anthrazyklins, Alter des Patienten, Bestrahlung u.a.) getroffen werden [51]. Die Autoren konnten anhand dieser Parameter zwischen Patienten mit hohem Risiko (57\% kardiale Ereignisse innerhalb von 5 Jahren) und niedrigem Risiko unterscheiden. Ein entsprechendes Screening aller Patienten erscheint jedoch (noch) nicht praktikabel.

\section{Chemotherapie-induzierte Polyneuropathie (CIPN)}

Die CIPN ist eine langanhaltende Komplikation, die oft bereits während der Krebstherapie auftritt und für Patienten sehr belastend ist. Eine nichtreversible CIPN schränkt die Lebensqualität ein und kann zu Berufsunfähigkeit führen. CIPN treten vorwiegend unter Platin- und Taxan-haltigen Therapien auf, aber auch bei Gabe von Vincristin, Bortezomib oder Thalidomid. Das Auftreten einer CIPN korreliert mit der kumulativ aufgenommen Dosis und manifestiert sich meist ab dem dritten Therapiezyklus. Die Inzidenz wird je nach Studie mit bis zu 38\% angegeben, wobei etwa 80\% der CIPN innerhalb von 6-8 Monaten nach Therapieende reversibel sind. Die Rückbildung kann sehr langsam erfolgen und in Einzelfällen mehr als 4 Jahre dauern. Besonders davon betroffen sind Patienten, die mit Cisplatin, Bleomycin und Vinblastin (Therapie bei Keimzelltumoren) behandelt wurden. Sie haben oft anhaltende Beschwerden für bis zu 10 Jahre [52-55]. Unter Therapie mit Oxaliplatin wird eine Persistenz der CIPN von fast $80 \%$ nach 29 Monaten angegeben, darunter ein beträchtlicher Anteil an höhergradigen Neuropathien [56]. In anderen Studien werden teils niedrige Persistenzraten genannt $[57,58]$, was mit der Verwendung unterschiedlicher Messinstrumente zur Erfassung einer CIPN zusammenhängen dürfte. Da es derzeit noch keine Standarddiagnostik auf CIPN gibt, ist ein Vergleich der Studienergebnisse schwierig.

Neben einer Reihe von physikalischen Therapien einschließlich Ergotherapie (Abb. 15) gibt es verschiedene medikamentöse Therapieoptionen bei CIPN.

In einer aktuellen Therapieempfehlung wird explizit auf die Prävention und Therapie der CIPN in der Gruppe der Langzeit-

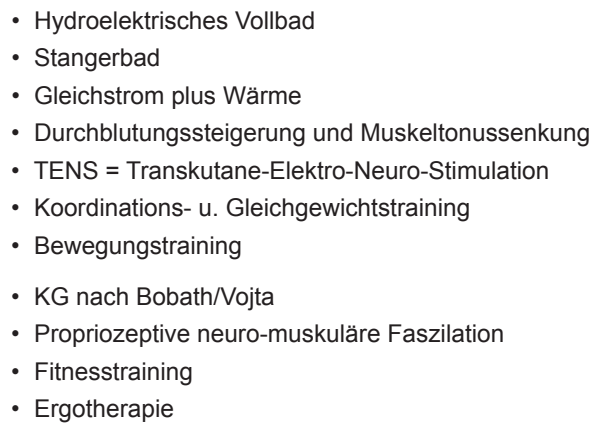

Abb. 15. Nichtmedikamentöse Therapien bei CIPN.

Krebs-Survivors eingegangen [59, 60]. Dabei handelt es sich allerdings im Wesentlichen um eine Listung der Substanzen, deren Effektivität bei CIPN nachweislich gering ist und deren Verwendung deshalb nicht sinnvoll ist. Dies gilt z.B. für Wachstumsfaktoren, Vitamine und Magnesium/Kalzium-Präparate. Eine positive Empfehlung aufgrund ausreichender Evidenz hat lediglich das Antidepressivum Duloxetin erhalten, das gegen Schmerzen bei diabetischer Polyneuropathie wirksam ist und in dieser Indikation auch zugelassen ist. Ob die Befunde nur auf CIPN übertragbar sind, wird derzeit von uns im Rahmen einer Fallserie untersucht.

Auch bei CIPN scheint es möglich zu sein, Patienten mit erhöhtem Risiko für eine schwere Oxaliplatin-induzierte Polyneuropathie mit Hilfe von SNPs zu identifizieren [61]. Eine ausgeprägte CIPN war bei Patienten mit einer Kombination relevanter SNP am wahrscheinlichsten.

\section{Gefahr der Infertilität sollte angesprochen werden}

Eine mögliche Infertilität nach Krebstherapie ist für Patienten im gebär- und zeugungsfähigen Alter eines der wichtigsten Themen überhaupt, wird aber dennoch eher zögerlich in der Beratung angesprochen. Dies gilt womöglich eher noch für junge Frauen als für junge Männer, denen mit der Kryokonservierung von Spermien immerhin eine Option angeboten werden kann. Die möglichen Schädigungen reichen von der temporären Amenorhö bis hin zur Infertilität, wobei etwa bei Chemotherapie nach dem BEACOPP-Regime die Infertilitätsrate bis zu 50\% beträgt (Abb. 16).

Außer den genannten Substanzen scheinen noch weitere bei Leukämie verwendete Wirkstoffe potentiell genotoxisch zu sein [63]; nähere Informationen finden sich unter dem Stichpunkt Fertilität auf der Homepage des Kompetenznetzes Leukämie.

Zur Verhinderung einer Ovarialinsuffizienz, die außer Infertilität auch Osteoporose und Beschwerden in der Menopause auslösen kann, wurden im Rahmen von Studien GnRH-Agonisten eingesetzt $[64,65]$. Sie zeigten bezüglich der Ovarprotektion unterschiedliche Ergebnisse, auch fehlten bisher Angaben zur Rate erfolgreich ausgetragener Schwangerschaften. Für die Subgruppe der Patientinnen mit Östrogenrezeptor-positivem Mammakarzinom konnte allerdings ein überzeugender Schutzeffekt der GnRH-Analoga auf die Ovarien nachgewiesen werden, was sich in einer Meta- 


\begin{tabular}{|c|c|c|}
\hline & 6 & 9 \\
\hline Chemotheraple & $\begin{array}{l}\text { Zerstörung des germinalen Epithels, } \\
\text { weniger der Leydig-und Sertoli- } \\
\text { Zellen } \\
\text { Altersabhăngige Schädigung höher } \\
\text { vor der Pubertăt } \\
\text { Hohes Risiko einer prolongierten } \\
\text { Azoospermie nach Alkyanzien- } \\
\text { oder Cisplatingabe, geringer nach } \\
\text { Vinkaalkaloiden, Bleomycin oder } \\
\text { Methotrexat }\end{array}$ & $\begin{array}{l}\text { Zerstörung der tellungsfáhigen } \\
\text { Zellen von Granulosa- und Tekazell- } \\
\text { schicht des Ovars } \\
\text { Altersabhängige dauerhafte oder } \\
\text { temporäre Amenorrhö } \\
\text { Vorzeitige Menopause } \\
\text { Vor allem nach Alkylanzientherapie, } \\
\text { aber auch nach Gabe anderer Sub- } \\
\text { stanzen (Doxorubicin, Cytarabin, } \\
\text { Cisplatin, Etoposid, Nitrosoharnstoffe, } \\
\text { Vinblastin) } \\
\text { Nach BEACOPP in ca. 50\% }\end{array}$ \\
\hline Radiotherapie & $\begin{array}{l}\text { Schădigung des Keimzellepithels, } \\
\text { nicht der Leydig-Zellen } \\
\text { Altersabhăngige Schădigung } \\
\text { Reduktion der Spermienzahl ab } \\
0,1 \text { Gy } \\
\text { Langzeitazoospermie ab 2-3 Gy } \\
\text { Anhaltende Infertilităt ab 6 Gy }\end{array}$ & $\begin{array}{l}\text { Direkte Schädigung der Oozyten } \\
\text { Altersabhăngige dauerhafte oder } \\
\text { temporăre Amenorrhö } \\
\text { Vorzeitige Menopause } \\
\text { Anhaltende ovarielle Funktionsein- } \\
\text { schränkung ab 4 Gy } \\
\text { Sterilität ab 14-20 Gy }\end{array}$ \\
\hline Operation & $\begin{array}{l}\text { Erektile Dysfunktion } \\
\text { Kosmetische Verstümmelung }\end{array}$ & Kosmetische Verstümmelung \\
\hline
\end{tabular}

Abb. 16. Therapieinduzierte Infertilität und Störungen der Sexualfunktionen (Auszug aus [63]).

Analyse bestätigt hat (57\%ige Reduktion für ovarielle Insuffizienz). Unterstützt werden diese Befunde durch eine aktuelle Phase-IIIStudie [66], allerdings in Bezug auf das Hormonrezeptor-negative Mammakarzinom. Die Studie untersuchte bei 218 prämenopausalen Patientinnen mit diesem Tumorbefund die ovarprotektive Wirkung von Goselerin in Verbindung mit einer Cyclophosphamid-haltigen Chemotherapie. Im Vergleich zu der nicht mit Goselerin behandelten Kontrollgruppe war in der Verumgruppe die Rate an Ovarialinsuffizienzen geringer (8 vs. 22\%) und es gab mehr gewollte Schwangerschaften ( 21 vs. $11 \%$ ) bei einer vergleichbaren Rate an Fehlgeburten, elektiven Entbindungen und Schwangerschaftskomplikationen.

Eine Übersicht über die Möglichkeiten zur Prävention und Therapie der Infertilität findet sich bei [67] sowie in modifizierter Form in den Online-Informationen des Kompetenznetzes Leukämie. Patienten und Ärzte können sich auch über die Homepage von FertiPROTEKT (www.fertiprotekt.de) über die Hintergründe und Behandlungenoptionen sowie deren Kosten informieren.

\section{Nachsorgenetzwerke in der Pädiatrischen}

Onkologie - deutsche und europäische Erfahrungen

\section{Referent: Jörn D. Beck, Erlangen}

Pro Jahr erkranken in Deutschland etwa 1800 Kinder und Jugendliche (im Alter bis zu 15 Jahren) an Krebs [68]. Das 5-JahresÜberleben dieser Patienten hat sich stetig verbessert und beträgt heute bei der Akuten Lymphatischen Leukämie (ALL) etwa 90\% und bei Morbus Hodgkin über 95\%. Doch auch wenn die Mehrzahl der Kinder die Krebserkrankung mehr als 5 Jahre überleben und in diesem Sinne Survivors sind, hat eine Vielzahl von ihnen mit Spätfolgen der Erkrankung oder der Behandlung zu kämpfen (Abb. 17). Andere wiederum, hier sind z.B. ehemalige ALL-Patienten der niedrigen und mittleren Risikogruppe zu nennen, sind abgesehen von wenigen Ausnahmen gesellschaftlich voll integriert

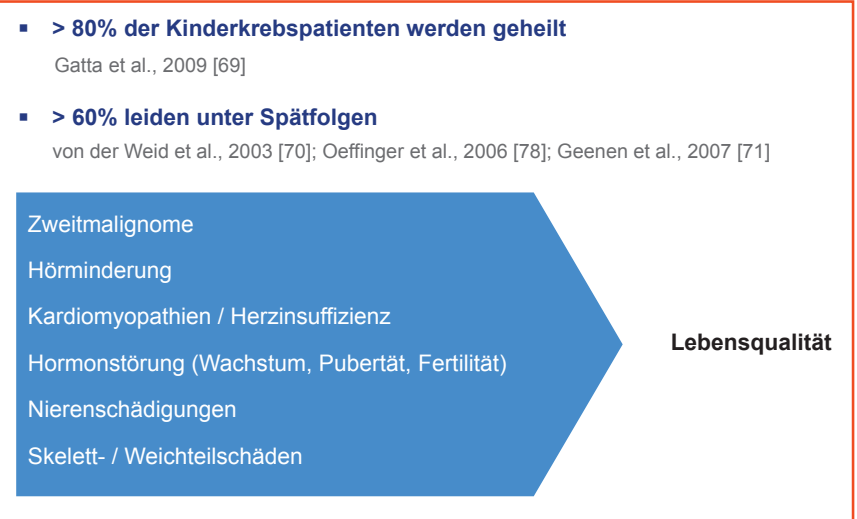

Abb. 17. Mögliche Spätfolgen nach Krebserkrankung im Kindesalter [69-71].

und führen ein Leben unbeeinträchtigt von der überstandenen Krebserkrankung und deren Behandlung.

Eine risikoadaptierte Nachsorge sollte diese Unterschiede nicht nur bei den mehr als 30000 ehemaligen Patienten in Deutschland berücksichtigen, sondern auch bei den 300000 bis 500000 Betroffenen in Europa, bei Bedarf lebenslang und in den Strukturen der Erwachsenenmedizin (Transition).

Diese Spätfolgen können die Lebensqualität noch Jahre nach der Krebstherapie nachhaltig beeinträchtigen. Von hoher Relevanz auch in Bezug auf die weitere Lebenserwartung sind Zweitmalignome. Nach Auswertung von ALL-BFM-Studien mit rund 5000 Kindern betrug das Risiko, nach erfolgreicher ALL-Therapie innerhalb von 15 Jahren erneut an Krebs zu erkranken, bei Kindern ohne ZNS-Bestrahlung 1,2\% und nach ZNS-Bestrahlung 3,5\% [72]. Das Risiko für Hirntumoren betrug ohne Bestrahlung 0,1\% und nach Bestrahlung $1,3 \%$.

Zur Chemoprophylaxe des Gehirns wird Methotrexat eingesetzt, in den 1970er Jahren kombiniert mit einer Bestrahlung von 24 Gy, die in den 1980er Jahren auf 12-18 Gy gesenkt werden konnte. Nach dieser Behandlung können häufiger morphologische Gehirnveränderungen und neurophysiologische und psychometrische Störungen nachgewiesen werden als bei einer ZNS-Prophylaxe, die ausschließlich Methotrexat nutzte [74]. Mit modernen Bestrahlungsgeräten und Techniken kann ungleich gewebeschonender bestrahlt werden.

Erkranken Mädchen im Alter $\geq 9$ Jahren an einem Morbus Hodgkin, haben sie aufgrund der Radiotherapie des Brustbereichs ein deutlich erhöhtes Risiko für Mammakarzinome [74] und sind deshalb beim Brustkrebs-Screening als Hochrisiko-Patientinnen einzuordnen.

Anfang der 1990er Jahre wurde in der Gesellschaft für Pädiatrische Onkologie und Hämatologie $(\mathrm{GPOH})$ ein Konzept zur risikoadaptierten, studienübergreifenden Nachsorge von Kindern mit Krebserkrankungen entwickelt, das wegweisend auch für andere Länder war [75]. Zur Erfassung und Verlaufskontrolle der Spätfolgen wird eine Standard-Basisdiagnostik eingesetzt, die bei Bedarf erweitert werden kann, etwa im Bereich Endokrinologie durch Hormonbestimmungen und Funktionstests (Abb. 18). 


\begin{tabular}{|c|c|c|}
\hline & Basic Methods & Detailed Methods \\
\hline \multicolumn{3}{|c|}{ - Physical examination } \\
\hline - CNS/PNS & $\begin{array}{l}\text { Neurologic examination } \\
\text { (esp. fine motor skills, } \\
\text { coordination) }\end{array}$ & $\begin{array}{l}\text { neuropsychological tests } \\
\text { CCT/MRI } \\
\text { velocity of nerve conduction }\end{array}$ \\
\hline $\begin{array}{l}\text { - Endocrine } \\
\text { system }\end{array}$ & $\begin{array}{l}\text { Auxology: height, weight, } \\
\text { (growth chart) } \\
\text { Pubertal development } \\
\text { (Tanner stages) }\end{array}$ & $\begin{array}{l}\text { Hormone levels } \\
\text { (cortisol, IGF1, IGFBP3 } \\
\text { TSH, fT4, LH, FSH, } \\
\text { estradiol or testosterone) } \\
\text { Endocrine function tests } \\
\text { Semen analysis }\end{array}$ \\
\hline - Hearing & Audiometry & Oto-acustic emissions \\
\hline - Heart & ECG, echocardiography & $\begin{array}{l}\text { Long-term ECG, exercise ECG } \\
\text { Radionuclide ventriculography }\end{array}$ \\
\hline
\end{tabular}

Abb. 18. LESS-Konzept zur Überwachung von Spätfolgen.

LESS(Late Effects Surveillance System)-Studienzentrum bündelt Erfahrungen mit Spätfolgen nach Krebs

Eine mögliche Spätfolge nach kranialer Strahlentherapie sind Störungen der Pubertätsentwicklung in Form einer Pubertas praecox (nach niedriger Strahlendosis im Rahmen einer prophylaktischen ZNS-Bestrahlung bei ALL) oder einer Pubertas tarda bei Gonadotropin-Mangel nach höheren Strahlendosen [76], die auch einen therapiebedürftigen Wachstumshormonmangel auslösen können.

Des Weiteren kann es zu Infertilität und Tubolopathien bei Kindern kommen, die eine Chemotherapie mit Alkylanzien erhalten haben. Treten diese oder andere Komplikationen auf, können sich die Betroffenen bzw. deren betreuende Ärzte an das LESS-Studienzentrum am Universitätsklinikum Lübeck wenden (thorsten. langer@uksh.de). Das ehemals in Erlangen gegründete Zentrum sammelt und analysiert Daten über Spätfolgen bei Überlebenden von Krebserkrankungen im Kindesalter innerhalb eines gut strukturierten Netzwerks. Dieses Netzwerk erfasst sämtliche Informationen über Spätfolgen, und zwar sowohl solche, die von den klinischen Studienleitungen direkt an das System gegeben werden, als auch Spätfolgen, die von den regionalen Kliniken, Fachärzten oder Hausärzten an das LESS-System gemeldet werden.

Die im LESS-Studienzentrum empfohlenen Nachsorgeuntersuchungen werden zurzeit überarbeitet, auch mithilfe der im europäischen PanCare-Konsortium entwickelten, evidenzbasierten Empfehlungen.

\section{Weitere Initiativen zur Erfassung von Spätkomplikationen}

Außer dem bereits erwähnten LESS werden Daten zum Outcome bei pädiatrischen Krebspatienten auch von weiteren Einrichtungen gesammelt und ausgewertet. So werden im 1981 aufgelegten Kinderkrebsregister in Mainz (www.kinderkrebsregister.de) nicht nur die Eckdaten fast aller pädiatrisch-onkologischen Patienten erfasst, sondern auch die Rate an Zweitmalignomen dokumentiert. Neben anderen Projekten werden die Adressen ehemaliger Patienten aktualisiert und in kontrollierter Weise für Untersuchungen zur Verfügung gestellt, so für das unter Leitung von Frau
G. Calaminus von der Krebshilfe geförderte, gerade angelaufene VIVE-Projekt. In Anlehnung an die amerikanische Childhood Cancer Survivor Study werden Betroffene angeschrieben und zu Spätfolgen sowie ihrer Lebenssituation befragt. Diese Gruppe umfasst ehemalige Krebspatienten im Alter von 25 bis 45 Jahren. Information: gabriele.calaminus@ukmuenster.de.

Darüber hinaus gibt es das Register zur Erfassung Strahlentherapie-bedingter Spätfolgen bei Kindern und Jugendlichen (RISK). Ziel dieses Registers ist es, bei Kindern, die im Rahmen einer Therapieoptimierungsstudie der GPOH im deutschsprachigen Raum strahlentherapeutisch behandelt werden, eine einheitliche Dokumentation der Bestrahlung sowie eine einheitliche, prospektive Erfassung der Nebenwirkungen zu gewährleisten. Information: Diana Steinmann (Steinmann.Diana@mh-hannover.de)

Demnach kooperieren im Nachsorgenetzwerk der GPOH vier Hauptstandorte mit den Studienleitungen in Lübeck (LESS), Münster (Lebensqualität und VIVE), Hannover (RISK) und Mainz (Deutsches Kinderkrebsregister) in der Arbeitsgruppe Langzeitbeobachtung.

\section{Lebenserwartung nach überstandener Krebserkrankung}

Die meisten Kinder mit Krebs überstehen zwar die Akuterkrankung für mindestens 5 Jahre und sind damit als Survivors zu betrachten, einige haben aber eine deutlich verkürzte Lebenserwartung [77]. Grund dafür ist unter anderem ein erhöhtes Risiko für Kardiovaskulopathien, wie ein Vergleich von über 10000 Überlebenden mit ihren Geschwistern in der amerikanischen Childhood Cancer Survivor Study gezeigt hat [78]. Insbesondere Anthrazyklin-Therapien können das Herz schädigen. Gefährdet sind besonders jüngere Kinder, die eine Thoraxbestrahlung sowie Anthrazykline erhalten haben. Die Schädigung ist nicht immer dosisabhängig [79]. Die Empfehlungen der Children's Oncology Group zur risikoadaptierten Sekundärprävention nach AnthrazyklinTherapie [80] werden bei der Erarbeitung von europäischen Nachsorgeempfehlungen berücksichtigt.

Es ist noch unklar, ob mit zunehmendem Alter der Betroffenen die in den frühen Behandlungsstudien eingesetzten hohen Anthrazyklin-Dosen als Risikofaktor gewertet werden müssen, ebenso wie die morphologischen Gehirnveränderungen nach ZNS-Prophylaxe einer ALL. In einer Untersuchung 25 Jahre nach Therapieende konnten Schädigungen der weißen Substanz, gekoppelt mit neuropsychologischen Einschränkungen, nachgewiesen werden [81].

An Krebs erkrankte Jugendliche müssen sich nicht nur mit ihrer Erkrankung und den dadurch hervorgerufenen Belastungen auseinandersetzen, sondern auch mit pubertätsbedingten Veränderungen. Die potentiell lebensbedrohliche Krankheit und die Nebenwirkungen der Therapie stehen den Bedürfnissen von Jugendlichen nach Unabhängigkeit und Neuorientierung entgegen. Dennoch verfügen die meisten Patienten über eine gute Resilienz und sind langfristig psychisch stabil. Bei einer nicht zu vernachlässigenden Subgruppe der Patienten liegt aber offenbar eine posttraumatische Belastungsstörung vor, wie in mehreren Studien gezeigt worden ist [82-85]. 


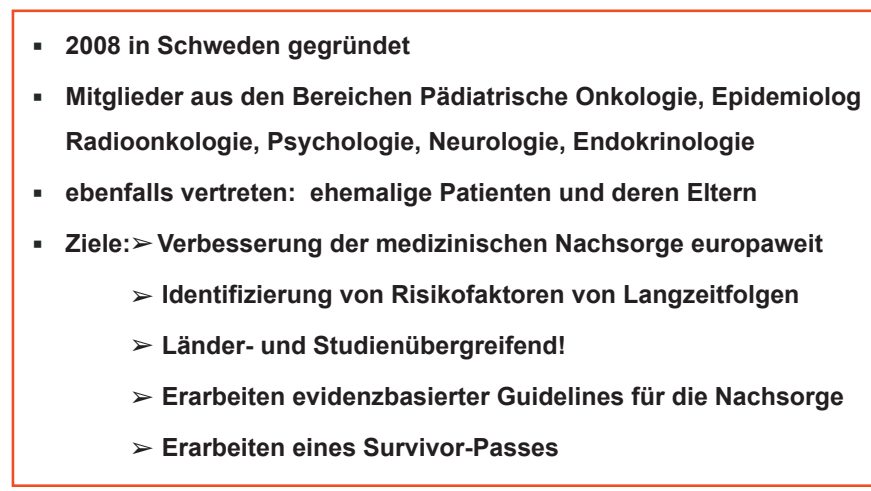

Abb. 19. Länderübergreifendes Projekt zur Nachsorge.

Pan-europäisches Netzwerk zur Reduktion von Spätfolgen

Auch das Pan-europäische Netzwerk zur Nachsorge und Reduktion von Spätfolgen nach einer Krebserkrankung im Kindes- und Jugendalter - PanCare - hat die Überlebenden einer Krebserkrankung im Kindes- und Jugendalter im Fokus. Ziel des Netzwerks ist es, allen Überlebenden in Europa eine optimale Versorgung zukommen zu lassen $[86,87]$. Dies geschieht unter Einbindung der Familienangehörigen (www.icccpo.org). PanCare wurde im Jahr 2008 gegründet, ist europaweit organisiert und vereinigt Experten aus verschiedenen Fachrichtungen (Abb. 19). Chairman ist Lars Hjorth von der Universität Lund, Schweden. Information: $w w w$. pancare.eu.

Zur besseren Information der nachsorgenden Ärzte und zur Stärkung der Autonomie ehemaliger Patienten wird im ebenfalls von der Europäischen Union geförderten ENCCA Netzwerk in Kooperation mit dem PanCare-Konsortium ein Survivor-Pass erarbeitet, in dem alle wichtigen, individuellen Informationen der Krebsbehandlung eines Patienten und die Nachsorgeempfehlungen enthalten sind (www.encca.eu). Aus dem PanCare-Netzwerk sind zwei Projekte hervorgegangen, die derzeit von der Europäischen Kommission (7. Rahmenprogramm) gefördert werden. Forscher und Institutionen aus 13 europäischen Ländern sind an den beiden Projekten PanCareSurFup und PanCareLife beteiligt [86]. Information: www.pancaresurfup.eu und www.pancarelife.eu.

\section{Teilprojekte: PanCareSurFup und PanCareLife}

Die im Jahr 2011 gestartete Studie PanCareSurFup erstellt eine europäische virtuelle Datenbank von Überlebenden, untersucht die "späte Mortalität», analysiert retrospektiv die strahlentherapeutische Dosimetrie und Chemotherapie mit kardiologischen Spätfolgen und Risiken eines Zweittumors und entwickelt evidenzbasierte Nachsorgeempfehlungen in Kooperation mit amerikanischen Kollegen $[87,88]$.

Die im November 2013 begonnene Studie PanCareLIFE (Abb. 20) untersucht nicht nur mit klinischen und epidemiologischen Daten die Fertilität und Ototoxizität (Hörminderung) unter dem Gesamtaspekt Lebensqualität nach Krebs im Kindes- und Jugendalter, sondern sucht auch nach einer genetischen Prädisposition für die Entstehung dieser Spätfolgen. So kann z.B. eine Behandlung mit Cisplatin bei einem Teil der Patienten die äußeren

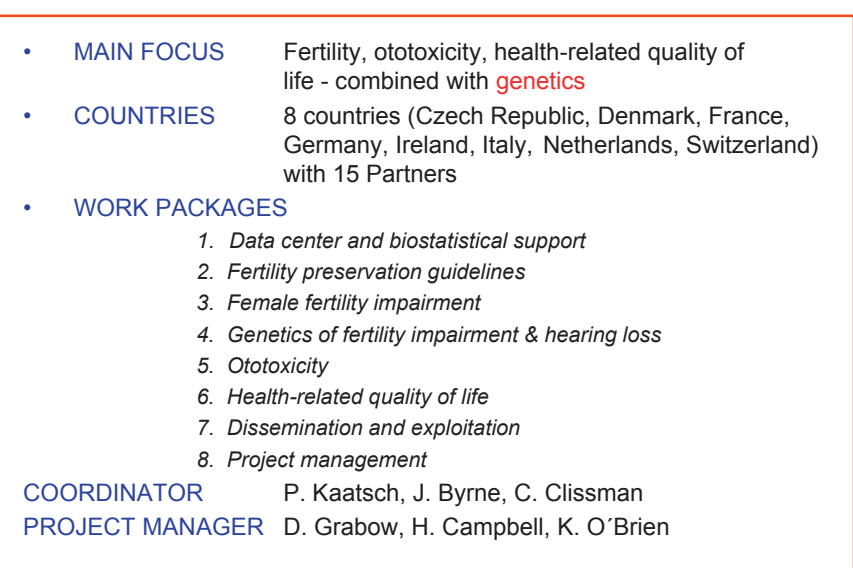

Abb. 20. PanCareLIFE greift auch genetische Fragestellungen auf.

Haarzellen im Innenohr schädigen und damit eine Hochtonschwerhörigkeit auslösen. Insbesondere Patienten mit bestimmten genetischen Polymorphismen scheinen ein erhöhtes Risiko für Hörschäden zu haben [89].

Zweimal jährlich werden PanCare-Meetings abgehalten. Hierzu sind Mitglieder sowie Interessenten herzlich eingeladen. Das nächste Treffen findet vom 23. bis 25. September 2015 in Wien vor dem dort stattfindenden Kongress der ECCO/ESMO statt.

Ohne die langjährige Zusammenarbeit mit den Kollegen T. Langer. P. Kaatsch, R. Skinner, R. Haupt, M. Jankovic, L. Hjorth und Frau G. Calaminus hätte ich die Arbeit nicht schreiben können. Ihnen danke ich für Ihre Unterstützung herzlich.

\section{Langzeitbetreuung - was bezahlen die Kassen?}

\section{Referentin: Barbara Zimmer, Düsseldorf}

Die onkologische Nachsorge hat zum Ziel, den Patienten bei seiner Gesundung zu unterstützen und die mit der Primärtherapie erreichten Erfolge zu sichern. Sie ermöglicht eine langfristige Begleitung des Patienten. Kurativ behandelbare Rezidive wie auch Folgen der Krebstherapie sollen frühzeitig erkannt werden.

Systematische, evidenzbasierte Nachsorgekonzepte und -empfehlungen in Leitlinien oder von Fachgesellschaften liegen vor (Abb. 21). Die Nachsorge wird individualisiert und risikoadaptiert umgesetzt. Dabei werden nicht nur die medizinischen Aspekte der Krebserkrankung berücksichtigt und dokumentiert, sondern auch jene der Lebensführung. Unterstützende Maßnahmen werden angesprochen. Eine gezielte Suche nach Fernmetastasen bei asymptomatischen Patienten ist nach derzeitiger Evidenzlage nicht sinnvoll.

\section{Evidenzgrundlagen der Nachsorge}

Der Einsatz der Maßnahmen in der Nachsorge sollte sich an der best-verfügbaren Evidenz orientieren und sich, soweit vorhanden, auf Ergebnisse randomisierter klinischer Studien stützen. Der für den Patienten relevante Nutzen muss stets größer sein als der Schaden, insbesondere bei Einsatz einer aufwendigen Diagnostik. Unter 


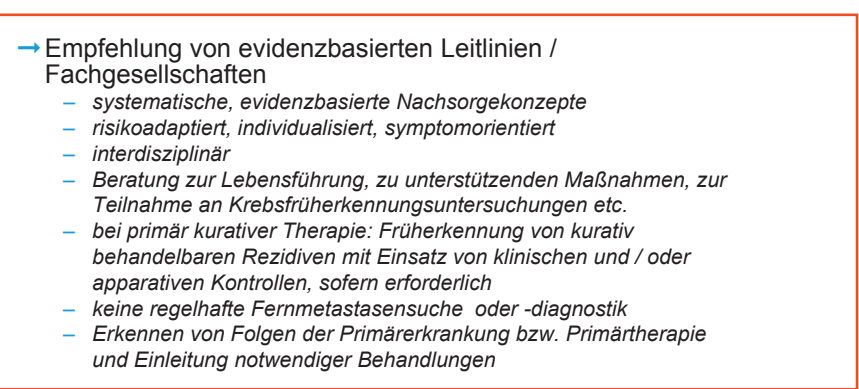

Abb. 21. Empfehlungen und Grundlagen zur Nachsorge.

diesem Gesichtspunkt spricht sich etwa die S3-Leitlinie zur Nachsorge bei Morbus Hodgkin gegen eine Routine-Computertomographie bei Patienten in kompletter Remission aus, bei denen kein Verdacht auf ein Rezidiv besteht und die beschwerdefrei sind [90] Kritisch sind auch regelmäßige Tumormarker-Kontrollen von $\mathrm{Pa}$ tientinnen mit Ovarialkarzinom zu sehen. Eine Studie hatte gezeigt, dass Frauen mit Ovarialkarzinom (FIGO I-IV) in kompletter Remission (nach einer platinbasierten Erstlinien-Chemotherapie) und normalisiertem CA125 von einer regelhaften Kontrolle des Tumormarkers nicht profitieren. Sie erhalten im Falle eines solchen laborchemischen Rezidivs zwar früher eine ZweitlinienChemotherapie, erreichen damit aber keinen Überlebensvorteil [91].

\section{Leistungsrechtliche Einordnung der onkologischen Nachsorge}

Die Nachsorge nach hämato-onkologischer bzw. onkologischer Behandlung ist begrifflich nicht zusätzlich gesondert im SGB V ausgewiesen. Die medizinisch notwendigen Maßnahmen sind Teil der kurativen Versorgung im Rahmen der der Krankenbehandlung (\$27 SGB V).

Die Leistungen nach $\$ 27$ SGB V umfassen unter anderem die ärztliche Behandlung, die Versorgung mit Arznei-, Verband-, Heil- und Hilfsmitteln, die Krankenhausbehandlung, die medizinische Rehabilitation und weitere Leistungen.

Die kurative Versorgung nach $\$ 27$ SGB V ist im Unterschied zur medizinischen Rehabilitation primär zentriert auf das klinische Bild als Manifestation einer Krankheit/Schädigung und ist a priori kausal orientiert. Ihr konzeptionelles Bezugssystem ist in der Regel das biomedizinische Krankheitsmodell und die entsprechende Klassifikation, die ICD. Sie unterscheidet sich darin vom biopsychosozialen Modell der Internationalen Klassifikation der Funktionsfähigkeit, Behinderung und Gesundheit (ICF), das in der Rehabilitation verwendet wird [92].

\section{Leistungsrechtliche Abgrenzungen}

Von den Leistungen zur onkologischen Nachsorge müssen die Leistungen nach $\$ 40-43$ SGB V abgegrenzt werden (Abb. 22). Sie können zum Teil zusätzlich in Anspruch genommen werden, wie etwa in Leistungen zur medizinischen Rehabilitation nach $₫ 40$ SGB V.

Medizinische Vorsorgeleistungen nach $\$ 23$ SGB V und $\$ 24$ SGB V (Medizinische Vorsorge für Mütter und Väter) sowie Gesundheitsuntersuchungen ( $\$ 25$ SGB V) und organisierte Früherken-

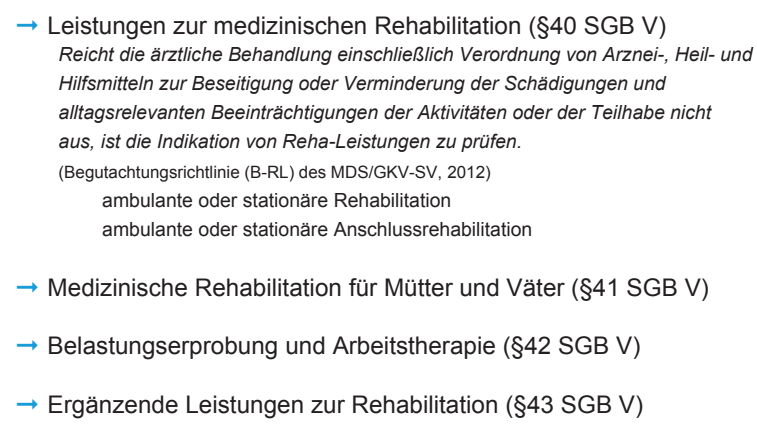

Abb. 22. Gesetzliche Regelungen im SGB V.

\section{DMP-Brustkrebs}

Strukturierte Behandlungsprogramme bei chronischen Krankheiten (§137c SGB V)

\section{Nachsorge: Umfang:}

mindestens Anamnese, körperliche Untersuchung

Aufklärung/Information

ist symptom- und risikoorientiert zu konzipieren

ist den individuellen Bedürfnissen der Patientin anzupassen

in der Regel halbjährlich plus erforderlichenfalls jederzeit

mindestens einmal jährlich Mammographie, ggf. häufiger

Psychosoziale Betreuung

Beratung über Möglichkeiten der Teilhabe/Rehabilitation

Prüfung, ob weitergehender Bedarf an Diagnostik oder Behandlung

\section{Körperliche Aktivitäten und Ernährung}

Beratung und Hinweise zu Maßnahmen der Eigenverantwortung

Abb. 23. Nachsorge im DMP-Brustkrebs.

nungsprogramme (\$25a SGB V) dienen der Prävention in der allgemeinen Bevölkerung, sind nicht der kurativen Nachsorge zuzurechnen. So hat eine Patientin mit Mammakarzinom Anspruch auf die notwendige ärztliche Betreuung und Behandlung innerhalb der Nachsorge der kurativen Versorgung; sie nimmt allerdings nicht parallel am Mammographie-Screening teil. Die gesetzlichen Leistungen zur Früherkennung von anderen Krebserkrankungen (z.B. Darmkrebs) stehen ihr dagegen offen.

Umfang und Ziele der Nachsorge sind im DMP-Brustkrebs, das Teil der strukturierten Behandlungsprogramme bei chronischen Krankheiten nach $\$ 137$ c SGB V ist, formuliert.

Das Ziel der Nachsorge ist die Unterstützung der physischen und psychischen Gesundung sowie der psychosozialen Rehabilitation [93]. Sie dient nicht nur der Verlaufskontrolle oder Nachbeobachtung der Erkrankung, sondern soll auch dazu beitragen, lokoregionale Rezidive und kontralaterale Tumoren sowie die Folgeerscheinungen der Primärtherapie frühzeitig zu erkennen und die Patientin in Maßnahmen der Eigenverantwortung zu unterstützen (Abb. 23).

\section{Leistungen der GKV}

Entsprechend der Zielsetzung, den Patienten bei seiner Gesundung zu unterstützen, können eine Reihe von Maßnahmen im Rahmen der Nachsorge umgesetzt werden. Dazu gehören: 
- Gespräche über bzw. Beratung zu

- Maßnahmen der Krankheitsbewältigung, der Eigenverantwortung, der Lebensführung und andere,

- Folgen der Erkrankung und der onkologischen Behandlung,

- unterstützenden Möglichkeiten, z.B. der Selbsthilfe,

- die Vermittlung bzw. Erwägung weiterer Maßnahmen wie die Verordnung von Hilfsmitteln, Arzneimitteln, Krankengymnastik, Rehabilitation, sozialmedizinischer Fallprüfung etc. sowie

- die Sicherung des Therapieerfolgs einschließlich adjuvanter Therapien und notwendiger Begleitbehandlungen.

Die vertraglichen/vertragsärztlichen Leistungen der GKV gemäß SGB V umfassen medizinisch notwendige Leistungen im Rahmen der Krankheitskontrolle (Nachbeobachtung):

- klinische und/oder apparative Kontrollen, evidenz- und leitlinienbasiert,

- frühzeitige (Lokal-)Rezidiverkennung bei kurativer Therapie (jedoch keine generelle Fernmetastasensuche/-diagnostik),

- Maßnahmen zum Erkennen und Behandeln von Folgen der Primärerkrankung und/oder -therapie, evidenz- und leitlinienbasiert.

Angesichts der Erfolge onkologischer Therapien mit Langzeitüberlebenden ergeben sich zunehmend auch Fragen hinsichtlich der Nachbeobachtung zu Spätfolgen einer Primärerkrankung und/ oder -therapie sowie zu möglichen Risiken von Sekundärmalignomen.

So konnte die systematische Langzeitnachbeobachtung von pädiatrischen Patienten der deutschen Hodgkin-Studiengruppe bei jungen Frauen, die im Kindes- oder Jungendalter wegen einer Erkrankung mit Morbus Hodgkin innerhalb der HD-78- bis HD90-Studie bestrahlt worden waren, eine erhöhte Rate an Brustkrebs nachgewiesen [74]. Von 590 Frauen, die im medianen Alter von 14 Jahren an Morbus Hodgkin erkrankt waren und eine Thoraxbestrahlung mit einer medianen Dosis von 30 Gy erhalten hatten, waren nach einem medianen Follow-up von fast 18 Jahren nur 7 Frauen an einem Hodgkin-Rezidiv erkrankt, allerdings 26 Frauen an Brustkrebs. Die kumulative Brustkrebsinzidenz über ein Follow-up von bis zu 30 Jahren betrug 16\%. Die Frauen waren zum Zeitpunkt der Brustkrebsdiagnose im Mittel 35 Jahre alt bei einem Intervall von etwa 21 Jahren zwischen Erst- und Zweitmalignom. In der Literatur sind ähnlich hohe Brustkrebsrisiken bei Frauen mit Hodgkin-Lymphom im Kindesalter ( $\geq 9$ Jahre) beschrieben [94]. Vor diesem Hintergrund sind Konzepte mit Empfehlungen zur Brustkrebs-Früherkennung bei Frauen mit Strahlentherapie im Thoraxbereich im Kindes-/Jugendalter publiziert [95].

Angesichts ihrer Erfahrungen erarbeitete die GPOH in Kooperation mit dem Deutschen Konsortium für Familiären Brust- und Eierstockkrebs ein Konzept für Früherkennungsuntersuchungen für Frauen mit hohem Brustkrebsrisiko nach Hodgkin-Lymphom im Kindes- und Jugendalter [94]. Das Kompetenz-Centrum Onkologie war daran beratend beteiligt, auch angesichts leistungsrechtlicher Fragen, die mit dem Spitzenverband Bund der Gesetzlichen Krankenkassen zu klären waren.

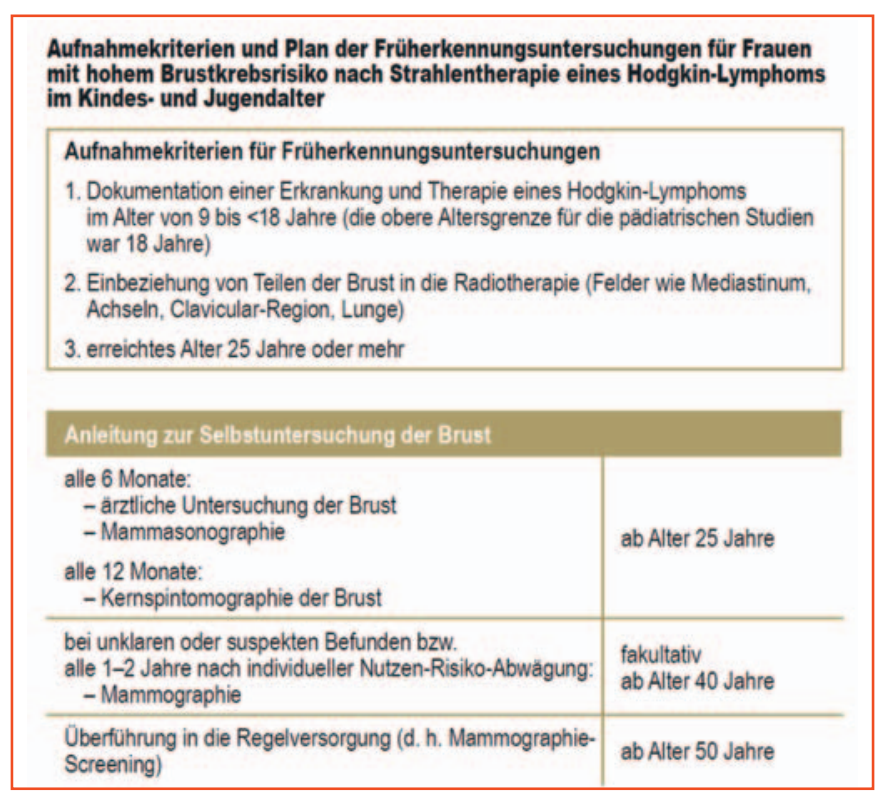

Abb. 24. Brustkrebs-Früherkennung bei erhöhtem Risiko nach Morbus Hodgkin (modifiziert nach [74]).

Empfohlen ist, für diese jungen Frauen, die im Konzept beschriebenen Leistungen (Abb. 24) im begründeten Einzelfall nach sorgfältiger Aufklärung bereitzustellen. Die Früherkennungsuntersuchungen werden an den Zentren des Deutschen Konsortiums für Familiären Brust- und Eierstockkrebs vorgenommen und in Kooperation mit der GPOH wissenschaftlich evaluiert.

Bisher konnten im Rahmen dieses Früherkennungskonzepts bei 7 von 98 untersuchten Frauen eine Brustkrebserkrankung identifiziert werden (vorläufige Daten; freundlicherweise zur Verfügung gestellt von Frau PD Dr. Rhiem, Zentrum für Familiären Brustund Eierstockkrebs, Köln, in Kooperation mit der GPOH). Weitere prospektive Auswertungen sind abzuwarten, auch um offene Fragen in der Umsetzung oder zu Nutzen und Schaden des Konzepts weiter zu klären.

\section{Onkologische Nachsorge - neue Konzepte}

Die medizinisch notwendigen Maßnahmen in der onkologischen Nachsorge sind Leistungen der kurativen Versorgung der GKV gemäß SGV V. Die Nachsorge von Patienten nach einer Krebserkrankung sollte evidenzbasiert, leitliniengestützt und risikoadaptiert erfolgen. In der Nachsorge werden notwendige Leistungen der vertragsärztlichen/vertraglichen Diagnostik und Therapie bereitgestellt, Bei Bedarf können auch weitere Maßnahmen oder gegebenenfalls Leistungen anderer Kostenträger, wie etwa der DRV, zum Tragen kommen.

Für die Entwicklung neuer Konzepte in der Nachsorge, wie dargestellt am Beispiel zu Früherkennungsuntersuchungen von Brustkrebs bei Langzeitüberlebenden nach Hodgkin-Lymphom im Kindes-/Jugendalter, bedarf es evidenzbasierter und versorgungsrelevanter Grundlagen, die eine medizinische Notwendigkeit und eine günstige Nutzen-Schaden-Abwägung erwarten lassen. Besondere Anforderungen - auch hinsichtlich der Leistungsbereitstellung 
durch die GKV - sind zu beachten. Die wissenschaftlich begleitete Umsetzung durch geeignete bzw. spezialisierte Erbringer wird empfohlen, damit der Stellenwert solcher neuen Konzepte hinsichtlich der Versorgung der Patientinnen und Patienten evaluiert werden kann.

\section{Disclosure Statement}

Bei Eva-Maria Kalusche-Bontemps, Pia Heußner, Ulf Seifart, Georgia Schilling und Barbara Zimmer bestehen keine Interessenkonflikte. Monika ReussBorst erhielt Forschungsunterstützung von der Deutschen Rentenversicherung Baden-Württemberg. Jörn D. Beck erhält Forschungsunterstützung vom Universitätsklinikum Lübeck.

\section{Literatur}

1 Deutsche Rentenversicherung: Sicherheit für Generationen. www.deutsche-rentenversicherung.de/Allgemein/ de/Navigation/5_Services/03_broschueren_und_mehr/ fachliteratur/statistiken_node.html (letzter Zugriff 5 . Mai 2015).

2 Deutsche Rentenversicherung Bund (Hrsg): Rentenversicherung in Zeitreihen 2014, DRV-Schriften Bd. 22, Berlin, Deutsche Rentenversicherung, 2014.

3 Deutsche Rentenversicherung Bund (Hrsg): Anforderungsprofil für eine stationäre Einrichtung zur onkologischen Rehabilitation Erwachsener. www.deutscherentenversicherung.de/cae/servlet/contentblob/226542/ publicationFile/16421/onko_reha_erwachsene.pdf(letzter Zugriff 16. Juni 2015)

4 Deutsche Rentenversicherung Bund (Hrsg): KTL-Klassifikation therapeutischer Leistungen in der medizinischen Rehabilitation, Berlin, Deutsche Rentenversicherung, 2015.

5 Deutsche Rentenversicherung Bund (Hrsg): Strukturqualität von Reha-Einrichtungen - Anforderungen der Deutschen Rentenversicherung Bund. Berlin, Deutsche Rentenversicherung, 2014.

6 DIMDI - Deutsches Institut für Medizinische Dokumentation und Information (Hrsg): Internationale Klassifikation der Funktionsfähigkeit, Behinderung und Gesundheit (Stand 2005). www.dimdi.de/static/de/ klassi/icf/ (letzter Zugriff 5. Mai 2015).

7 Deutsche Rentenversicherung Bund (Hrsg): Arbeitsbuch Reha-Ziele Zielvereinbarungen in der medizinischen Rehabilitation, Berlin, Deutsche Rentenversicherung, 2014.

8 Deutsche Rentenversicherung Bund (Hrsg): Sozialmedizinisches Glossar im Internet: www.deutsche-rentenversicherung.de/Allgemein/de/Inhalt/3_Infos_fuer Experten/01_sozialmedizin_forschung/01_sozialmedizin/ 08_sozmed_glossar/Functions/Glossar.html?cms_lv2= 238992ఓ cms_lv3=217732 (letzter Zugriff 5. Mai 2015).

9 Deutsche Rentenversicherung Bund (Hrsg): Leitlinien für die sozialmedizinische Begutachtung. Leistungsfähigkeit bei Mamma-Karzinom. Berlin, Deutsche Rentenversicherung, 2011.

10 Huy C, Schmidt ME, Vrieling A, Chang-Claude J, Steindorf K: Physical activity in a German breast cancer patient cohort: one-year trends and characteristics associated with change in activity level. Eur J Cancer 2012;48:297-304.

11 Newsom J, Estrada CA, Panisko D, Willett L: Selecting the best clinical vignettes for academic meetings: should the scoring tool criteria be modified? J Gen In tern Med 2012;27:202-206.

12 Brown JC, Winters-Stone K, Lee A, Schmitz KH: Cancer, physical activity, and exercise. Compr Physiol 2012;2:2775-2809.

13 Speck RM, Courneya KS, Mâsse LC, Duval S, Schmitz $\mathrm{KH}$ : An update of controlled physical activity trials in cancer survivors: a systematic review and meta-analysis. J Cancer Surviv 2010;4:87-100.

14 Craft LL, Vaniterson EH, Helenowski IB, Rademaker AW, Courneya KS: Exercise effects on depressive symptoms in cancer survivors: a systematic review and meta-analysis. Cancer Epidemiol Biomarkers Prev 2012;21:3-19.
15 Cheema BS, Kilbreath SL, Fahey PP, Delaney GP, Atlantis E: Safety and efficacy of progressive resistance training in breast cancer: a systematic review and metaanalysis. Breast Cancer Res Treat 2014;148:249-268.

16 Schmitz KH, Courneya KS, Matthews C, DemarkWahnefried W, Galvão DA, Pinto BM, Irwin ML, Wolin KY, Segal RJ, Lucia A, Schneider CM, von Gruenigen VE, Schwartz AL; American College of Sports Medicine: American College of Sports Medicine roundtable on exercise guidelines for cancer survivors. Med Sci Sports Exerc 2010;42:1409-1426. Erratum in: Med Sci Sports Exerc 2011;43:195

17 Andersen BL, Yang HC, Farrar WB, Golden-Kreutz DM, Emery CF, Thornton LM, Young DC, Carson WE 3rd: Psychologic intervention improves survival for breast cancer patients: a randomized clinical trial. Cancer 2008;113:3450-3458.

18 Faller H, Schuler M, Richard M, Heckl U, Weis J, Küffner R: Effects of psycho-oncologic interventions on emotional distress and quality of life in adult patients with cancer: systematic review and meta-analysis. J Clin Oncol 2013;31:782-793.

19 Mewes JC, Steuten LM, Ijzerman MJ, van Harten WH: Effectiveness of multidimensional cancer survivor rehabilitation and cost-effectiveness of cancer rehabilitation in general: a systematic review. Oncologist 2012; 17:1581-1593.

20 Fuji S, Mori T, Khattry N, Cheng J, Do YR, Yakushijin K, Kohashi S, Fukuda T, Kim SW: Severe weight loss in 3 months after allogeneic hematopoietic SCT was associated with an increased risk of subsequent non-relapse mortality. Bone Marrow Transplant 2015;50:100-105.

21 Chlebowski RT, Blackburn GL: Final survival analyses from the Women's Intervention Nutrition Study (WINS) evaluation dietary fat reduction as adjuvant breast cancer therapy. SABCS 2014;abstract S5-08. www.abstracts2view.com/sabcs14/view.php?nu= SABCS13L_648 (letzer Zugriff 5. Mai 2015).

22 de Boer AG, Taskila T, Ojajärvi A, van Dijk FJ, Verbeek JH: Cancer survivors and unemployment: a meta-analysis and meta-regression. JAMA 2009;301:753-762.

23 Robert Koch-Institut (Hrsg): Beiträge zur Gesundheitsberichterstattung des Bundes. Krebs in Deutschland 2009/2010. www.rki.de/DE/Content/Gesundheitsmonitoring/Gesundheitsberichterstattung/GBEDownloadsB/KID2013.pdf;jsessionid=33A0FA47F32A680DA 700261C6F793ED7.2_cid290?_blob=publicationFile (letzter Zugriff 5. Mai 2015).

24 Mehnert A: Employment and work-related issues in cancer survivors. Crit Rev Oncol Hematol 2011;77: 109-130.

25 Luengo-Fernandez R, Leal J, Gray A, Sullivan R: Economic burden of cancer across the European Union: a population-based cost analysis. Lancet Oncol 2013;14: 1165-1174.

26 Delgado-Guay MO, Ferrer J, Rieber AG, Rhondali W, Ochoa J, Cantu H, Chisholm GB, Williams JL, FrisbeeHume S, Bruera E: Frequency, intensity, and correlates of financial distress (FD) among advanced cancer patients (AdCa). J Clin Oncol 2014;32(suppl): abstr 9635.
27 Albano JD, Ward E, Jemal A, Anderson R, Cokkinides VE, Murray T, Henley J, Liff J, Thun MJ: Cancer mortality in the United States by education level and race. J Natl Cancer Inst 2007;99:1384-1394.

28 Kroenke CH, Kubzansky LD, Schernhammer ES, Holmes MD, Kawachi I: Social networks, social support, and survival after breast cancer diagnosis. J Clin Oncol 2006;24:1105-1111.

29 Keegan TH, McClure LA, Foran JM, Clarke CA: Improvements in survival after follicular lymphoma by race/ethnicity and socioeconomic status: a populationbased study. J Clin Oncol 2009;27:3044-3051.

30 Kristinsson SY, Derolf AR, Edgren G, Dickman PW, Björkholm M: Socioeconomic differences in patient survival are increasing for acute myeloid leukemia and multiple myeloma in Sweden. J Clin Oncol 2009;27: 2073-2080.

31 Petridou ET, Sergentanis TN, Perlepe C, Papathoma P, Tsilimidos G, Kontogeorgi E, Kourti M, Baka M, Moschovi M, Polychronopoulou S, Sidi V, Hatzipantelis E, Stiakaki E, Iliadou AN, La Vecchia C, Skalkidou A, Adami HO: Socioeconomic disparities in survival from childhood leukemia in the United States and globally: a meta-analysis. Ann Oncol 2015;26:589-597.

32 Jansen L, Eberle A, Emrich K, Gondos A, Holleczek B, Kajüter H, Maier W, Nennecke A, Pritzkuleit R, Brenner $\mathrm{H}$; for the GEKID Cancer Survival Working Group: Socioeconomic deprivation and cancer survival in Germany: an ecological analysis in 200 districts in Germany. Int J Cancer 2014;134:2951-2960.

33 de Boer AG, Taskila T, Ojajärvi A, van Dijk FJ, Verbeek JH: Cancer survivors and unemployment: a meta-analysis and meta-regression. JAMA 2009;301:753-762.

34 Dowling EC, Chawla N, Forsythe LP, de Moor J, McNeel T, Rozjabek HM, Ekwueme DU, Yabroff KR: Lost productivity and burden of illness in cancer survivors with and without other chronic conditions. Cancer 2013;119:3393-3401.

35 Shankaran V, Jolly S, Blough D, Ramsey SD: Risk factors for financial hardship in patients receiving adjuvant chemotherapy for colon cancer: a population-based exploratory analysis. J Clin Oncol 2012;30:1608-1614.

36 Spelten ER, Verbeek JH, Uitterhoeve AL, Ansink AC, van der Lelie J, de Reijke TM, Kammeijer M, de Haes JC, Sprangers MA: Cancer, fatigue and the return of patients to work - a prospective cohort study. Eur J Cancer 2003;39:1562-1567.

37 Mehnert A, Koch U: Predictors of employment among cancer survivors after medical rehabilitation - a prospective study. Scand J Work Environ Health 2013;39:76-87.

38 Aleman BM, van den Belt-Dusebout AW, De Bruin ML, van't Veer MB, Baaijens MH, de Boer JP, Hart AA, Klokman WJ, Kuenen MA, Ouwens GM, Bartelink $\mathrm{H}$, van Leeuwen FE: Late cardiotoxicity after treatment for Hodgkin lymphoma. Blood 2007; 109: 1878-1886.

39 Von Hoff DD, Layard MW, Basa P, Davis HL Jr, Von Hoff AL, Rozencweig M, Muggia FM: Risk factors for doxorubicin-induced congestive heart failure. Ann Intern Med 1979;91:710-717. 
40 Van der Vijgh WJ, Maessen PA, Pinedo HM: Comparative metabolism and pharmacokinetics of doxorubicin and 4'-epidoxorubicin in plasma, heart and tumor of tumor-bearing mice. Cancer Chemother Pharmacol 1990;26:9-12.

41 Chello M, Mastroroberto P, Romano R, Castaldo P, Bevacqua E, Marchese AR: Protection by coenzyme Q10 of tissue reperfusion injury during abdominal aortic cross-clamping. J Cardiovasc Surg (Torino) 1996;37:229-235.

42 van der Pal HJ, van Dalen EC, van Delden E, van Dijk IW, Kok WE, Geskus RB, Sieswerda E, Oldenburger F, Koning CC, van Leeuwen FE, Caron HN, Kremer LC: High risk of symptomatic cardiac events in childhood cancer survivors. J Clin Oncol 2012;30:1429-1437.

43 Romond EH, Jeong JH, Rastogi P, Swain SM, Geyer CE Jr, Ewer MS, Rathi V, Fehrenbacher L, Brufsky A, Azar CA, Flynn PJ, Zapas JL, Polikoff J, Gross HM, Biggs DD, Atkins JN, Tan-Chiu E, Zheng P, Yothers G, Mamounas EP, Wolmark N: Seven-year follow-up assessment of cardiac function in NSABP B-31, a randomized trial comparing doxorubicin and cyclophosphamide followed by paclitaxel (ACP) with ACP plus trastuzumab as adjuvant therapy for patients with node-positive, human epidermal growth factor receptor 2-positive breast cancer. J Clin Oncol 2012;30:3792-3799.

44 Plana JC, Galderisi M, Barac A, Ewer MS, Ky B, Scherrer-Crosbie M, Ganame J, Sebag IA, Agler DA, Badano LP, Banchs J, Cardinale D, Carver J, Cerqueira M, DeCara JM, Edvardsen T, Flamm SD, Force T, Griffin BP, Jerusalem G, Liu JE, Magalhães A, Marwick T, Sanchez LY, Sicari R, Villarraga HR, Lancellotti P: Expert consensus for multimodality imaging evaluation of adult patients during and after cancer therapy: a report from the American Society of Echocardiography and the European Association of Cardiovascular Imaging. J Am Soc Echocardiogr 2014;27:911-939.

45 Curigliano G, Cardinale D, Suter T, Plataniotis G, de Azambuja E, Sandri MT, Criscitiello C, Goldhirsch A, Cipolla C, Roila F; ESMO Guidelines Working Group: Cardiovascular toxicity induced by chemotherapy, targeted agents and radiotherapy: ESMO Clinical Practice Guidelines. Ann Oncol 2012;23(suppl 7):vii155-166.

46 van Dalen EC, Michiels EM, Caron HN, Kremer LC: Different anthracycline derivates for reducing cardiotoxicity in cancer patients. Cochrane Database Syst Rev 2010;5:CD005006.

47 Swain SM, Vici P: The current and future role of dexrazoxane as a cardioprotectant in anthracycline treatment: expert panel review. J Cancer Res Clin Oncol 2004;130:1-7.

48 Bundesärztekammer (BÄK), Kassenärztliche Bundesvereinigung (KBV), Arbeitsgemeinschaft der Wissenschaftlichen Medizinischen Fachgesellschaften (AWMF): Nationale Versorgungsleitlinie Chronische Herzinsuffizienz - Kurzfassung, 1. Auflage. Version 7. 2009, zuletzt geändert: August 2013. www.leitlinien.de/ mdb/downloads/nvl/herzinsuffizienz/herzinsuffizienz1aufl-vers7-kurz.pdf (letzter Zugriff 5. Mai 2015).

49 Cardinale D, Colombo A, Lamantia G, Colombo N, Civelli M, De Giacomi G, Rubino M, Veglia F, Fiorentini C, Cipolla CM: Anthracycline-induced cardiomyopathy: clinical relevance and response to pharmaco logic therapy. J Am Coll Cardiol 2010;55:213-220.

50 Dessì M, Piras A, Madeddu C, Cadeddu C, Deidda M, Massa E, Antoni G, Mantovani G, Mercuro G: Longterm protective effects of the angiotensin receptor blocker telmisartan on epirubicin-induced inflammation, oxidative stress and myocardial dysfunction. Exp Ther Med 2011;2:1003-1009.
51 Visscher H, Ross CJ, Rassekh SR, Barhdadi A, Dubé MP, Al-Saloos H, Sandor GS, Caron HN, van Dalen EC, Kremer LC, van der Pal HJ, Brown AM, Rogers PC, Phillips MS, Rieder MJ, Carleton BC, Hayden MR; Canadian Pharmacogenomics Network for Drug Safety Consortium: Pharmacogenomic prediction of anthracycline-induced cardiotoxicity in children. J Clin Oncol 2012;30:1422-1428.

52 Argyriou AA, Bruna J, Marmiroli P, Cavaletti G: Chemotherapy-induced peripheral neurotoxicity (CIPN): an update. Crit Rev Oncol Hematol 2012;82:51-77.

53 Postma TJ, Benard BA, Huijgens PC, Ossenkoppele GJ, Heimans JJ: Long-term effects of vincristine on the peripheral nervous system. J Neurooncol 1993;15:23-37.

54 Postma TJ, Heimans JJ: Grading of chemotherapy-induced peripheral neuropathy. Ann Oncol 2000;11: 509-513.

55 Hansen SW: Late-effects after treatment for germ-cell cancer with cisplatin, vinblastine, and bleomycin. Dan Med Bull 1992;39:391-399.

56 Park SB, Lin CS, Krishnan AV, Goldstein D, Friedlander ML, Kiernan MC: Long-term neuropathy after oxaliplatin treatment: challenging the dictum of reversibility. Oncologist 2011;16:708-716.

57 Land SR, Kopec JA, Cecchini RS, Ganz PA, Wieand HS, Colangelo LH, Murphy K, Kuebler JP, Seay TE, Needles BM, Bearden JD 3rd, Colman LK, Lanier KS, Pajon ER Jr, Cella D, Smith RE, O'Connell MJ, Costantino JP, Wolmark N: Neurotoxicity from oxaliplatin combined with weekly bolus fluorouracil and leucovorin as surgical adjuvant chemotherapy for stage II and III colon cancer: NSABP C-07. J Clin Oncol 2007;25: 2205-2211.

58 André T, Boni C, Navarro M, Tabernero J, Hickish T, Topham C, Bonetti A, Clingan P, Bridgewater J, Rivera F, de Gramont A: Improved overall survival with oxaliplatin, fluorouracil, and leucovorin as adjuvant treatment in stage II or III colon cancer in the MOSAIC trial. J Clin Oncol 2009;27:3109-3116.

59 Hershman DL, Lacchetti C, Dworkin RH, Lavoie Smith EM, Bleeker J, Cavaletti G, Chauhan C, Gavin P, Lavino A, Lustberg MB, Paice J, Schneider B, Smith ML, Smith T, Terstriep S, Wagner-Johnston N, Bak K, Loprinzi CL; American Society of Clinical Oncology: Prevention and management of chemotherapy-induced peripheral neuropathy in survivors of adult cancers: American Society of Clinical Oncology clinical practice guideline. J Clin Oncol 2014;32:1941-1967.

60 Loprinzi CL, Qin R, Dakhil SR, Fehrenbacher L, Flynn KA, Atherton P, Seisler D, Qamar R, Lewis GC, Grothey A: Phase III randomized, placebo-controlled, doubleblind study of intravenous calcium and magnesium to prevent oxaliplatin-induced sensory neurotoxicity (N08CB/Alliance). J Clin Oncol 2014;32:997-1005.

61 Custodio A, Moreno-Rubio J, Aparicio J, Gallego-Plazas J, Yaya R, Maurel J, Higuera O, Burgos E, Ramos D, Calatrava A, Andrada E, López R, Moreno V, Madero R, Cejas P, Feliu J: Pharmacogenetic predictors of severe peripheral neuropathy in colon cancer patients treated with oxaliplatin-based adjuvant chemotherapy: a GEMCAD group study. Ann Oncol 2014;25:398-403.

62 Schilling G, Arnold D: Therapiefolgen bei Krebs-Langzeitüberlebenden. Bundesgesundheitsbl Gesundheitsforsch Gesundheitsschutz 2012;55:493-500.

63 Pereyra Pacheco B, Mendez Ribas IM, Milone G, Fernández I, Kvicala R, Mila T, Di Noto A, Contreras Ortiz O, Pavlovsky S: Use of GnRH analogs for functional protection of the ovary an preservation of fertility during cancer treatment in adolescents: a preliminary report. Gynecol Oncol 2001,81:391-397.
64 Del Mastro L, Boni L, Michelotti A, Gamucci T, Olmeo N, Gori S, Giordano M, Garrone O, Pronzato P, Bighin C, Levaggi A, Giraudi S, Cresti N, Magnolfi E, Scotto $\mathrm{T}$, Vecchio C, Venturini M: Effect of the gonadotropin-releasing hormone analogue triptorelin on the occurrence of chemotherapy-induced early menopause in premenopausal women with breast cancer: a randomized trial. JAMA 2011;20;306:269-276.

65 Del Mastro L, Levaggi A, Giraudi S, Pronzato P: Luteinising hormone releasing hormone agonists ( $\mathrm{LH}-$ $\mathrm{RHa}$ ) in premenopausal early breast cancer patients: current role and future perspectives. Cancer Treat Rev 2011;37:208-211.

66 Moore HC, Unger JM, Phillips KA, Boyle F, Hitre E, Porter D, Francis PA, Goldstein LJ, Gomez HL, Vallejos CS, Partridge AH, Dakhil SR, Garcia AA, Gralow J, Lombard JM, Forbes JF, Martino S, Barlow WE, Fabian CJ, Minasian L, Meyskens FL Jr, Gelber RD, Hortobagyi GN, Albain KS; POEMS/S0230 Investigators: Goserelin for ovarian protection during breast-cancer adjuvant chemotherapy. N Engl J Med 2015;372:923-932.

67 Orwig KE, Schlatt S: Cryopreservation and transplantation of spermatogonia and testicular tissue for preservation of male fertility. J Natl Cancer Inst Monogr 2005;(34):51-56.

68 Kaatsch P, Spix C: German Childhood Cancer Registry - Report 2013/14 (1980-2013). Mainz, Institute of Medical Biostatistics, Epidemiology and Informatics (IMBEI) at the University Medical Center of the Johannes Gutenberg University Mainz, 2014.

69 Gatta G, Zigon G, Capocaccia R, Coebergh JW, Desandes E, Kaatsch P, Pastore G, Peris-Bonet R, Stiller CA; EUROCARE Working Group: Survival of European children and young adults with cancer diagnosed 1995-2002.Eur J Cancer 2009;45:992-1005.

70 von der Weid N, Mosimann I, Hirt A, Wacker P, Nenadov Beck M, Imbach P, Caflisch U, Niggli F, Feldges A, Wagner HP: Intellectual outcome in children and adolescents with acute lymphoblastic leukaemia treated with chemotherapy alone: age- and sex-related differences.Eur J Cancer 2003;39:359-365.

71 Geenen MM, Cardous-Ubbink MC, Kremer LC, van den Bos C, van der Pal HJ, Heinen RC, Jaspers MW, Koning CC, Oldenburger F, Langeveld NE, Hart AA, Bakker PJ, Caron HN, van Leeuwen FE: Medical assessment of adverse health outcomes in long-term survivors of childhood cancer. JAMA 2007;297:2705-2715.

72 Löning L, Zimmermann M, Reiter A, Kaatsch P, Henze G, Riehm H, Schrappe M: Secondary neoplasms subsequent to Berlin-Frankfurt-Münster therapy of acute lymphoblastic leukemia in childhood: significantly lower risk without cranial radiotherapy. Blood 2000; 95:2770-2775.

73 Langer T, Huk WJ, Hertzberg H, Überal MA, Meier W, Korinthenberg R, Beck JD: Akute lymphoblastische Leukämie im Kindesalter: Morphe und Funktion nach Behandlungsende. Dtsch Arztebl 1998;95:A-3058 / B-2587 /C-2296.

74 Schellong G, Riepenhausen M, Ehlert K, Brämswig J, Dörffel W; German Working Group on the Long-Term Sequelae of Hodgkin's Disease, Schmutzler RK, Riehm K, Bick U; German Consortium for Hereditary Breast and Ovarian Cancer: Breast cancer in young women after treatment for Hodgkin's disease during childhood or adolescence - an observational study with up to 33year follow-up. Dtsch Arztebl Int 2014;111:3-9.

75 Beck JD, Winkler K, Niethammer D, Brandis M, Hertzberg H, von der Hardt K, Greil J, Uberall MA, Rossi R, Lamprecht-Dinnesen A, et al: After-care of children and young adults surviving cancer. Initial recommendations by the late sequelae study group (in German). Klin Padiatr 1995;207:186-192. 
76 Müller J: Disturbance of pubertal development after cancer treatment. Best Pract Res Clin Endocrinol Metab 2002;16:91-103.

77 Armstrong GT, Liu Q, Yasui Y, Neglia JP, Leisenring W, Robison LL, Mertens AC: Late mortality among 5-year survivors of childhood cancer: a summary from the Childhood Cancer Survivor Study. J ClinOncol 2009;27:2328-2338.

78 Oeffinger KC, Mertens AC, Sklar CA, Kawashima T, Hudson MM, Meadows AT, Friedman DL, Marina N, Hobbie W, Kadan-Lottick NS, Schwartz CL, Leisenring W, Robison LL; Childhood Cancer Survivor Study: Chronic health conditions in adult survivors of childhood cancer. N Engl J Med 2006;355:1572-1582.

79 Children's Oncology Group: Heart Health. Version 4.0. Oktober 2013. www.survivorshipguidelines.org (letzter Zugriff 11. Mai 2015).

80 Trachtenberg BH, Landy DC, Franco VI, Henkel JM, Pearson EJ, Miller TL, Lipshultz SE: Anthracyclineassociated cardiotoxicity in survivors of childhood cancer. Pediatr Cardiol 2011;32:342-353.

81 Schuitema I, Deprez S, Van Hecke W, Daams M, Uyttebroeck A, Sunaert S, Barkhof F, van Dulmen-den Broeder E, van der Pal HJ, van den Bos C, Veerman AJ, de Sonneville LM: Accelerated aging, decreased white matter integrity, and associated neuropsychological dysfunction 25 years after pediatric lymphoid malignancies. J Clin Oncol 2013;31:3378-3388.

82 Hobbie WL, Stuber M, Meeske K, Wissler K, Rourke MT, Ruccione K, Hinkle A, Kazak AE: Symptoms of posttraumatic stress in young adult survivors of childhood cancer.J Clin Oncol 2000;18:4060-4066.

83 Langeveld NE, Grootenhuis MA, Voûte PA, de Haan RJ: Posttraumatic stress symptoms in adult survivors of childhood cancer. Pediatr Blood Cancer 2004;42: 604-610.
84 Schwartz L, Drotar D: Posttraumatic stress and related impairment in survivors of childhood cancer in early adulthood compared to healthy peers. J Pediatr Psychol 2006;31:356-366.

85 Lee YL, Santacroce SJ: Posttraumatic stress in long-term young adult survivors of childhood cancer: a questionnaire survey.Int J Nurs Stud 2007;44:1406-1417.

86 Haupt R, Spinetta JJ, Ban I, Barr RD, Beck JD, Byrne J, et al: Long term survivors of childhood cancer: cure and care. The Erice Statement. Eur J Cancer 2007;43: 1778-1780.

87 Hjorth L, Haupt R, Skinner R, Grabow D, Byrne J, Karner S, Levitt G, Michel G, van der Pal H, Bárdi E, Beck JD, de Vathaire F, Essig S, Frey E, Garwicz S, Hawkins M, Jakab Z, Jankovic M, Kazanowska B, Kepak T, Kremer L, Lackner H, Sugden E, Terenziani M, Zadravec Zaletel L, Kaatsch P; on behalf of the PanCare Network: Survivorship after childhood cancer: PanCare: A European Network to promote optimal long-term care. Eur.J Cancer 2015; doi: 10.1016/j.ejca.2015.04.002.

88 Brown MC, Levitt GA, Frey E, Bárdi E, Haupt R, Hjorth L, Kremer L, Kuehni CE, Lettner C, Mulder RL, Michel G, Skinner R, on behalf of the PanCareSurFup Consortium: The views of European clinicians on guidelines for long-term follow-up of childhood cancer survivors. Eur J Cancer 2015;51:1203-1211.

89 Langer T, am Zehnhoff-Dinnesen A, Radtke S, Meitert J, Zolk O: Understanding platinum-induced ototoxicity. Trends Pharmacol Sci 2013;34:458-469.

90 Deutsche Krebsgesellschaft e.V., Deutsche Krebshilfe e.V. (Hrsg): Leitlinienprogramm Onkologie der AWMF: Hodgkin Lymphom. S3-Leitlinie Diagnostik, Therapie und Nachsorge des Hodgkin Lymphoms bei erwachsenen Patienten. Version 1.0, Februar 2013. AWMF Registernummer: 018/029OL.
91 Rustin GJ, van der Burg ME, Griffin CL, Guthrie D, Lamont A, Jayson GC, Kristensen G, Mediola C, Coens C, Qian W, Parmar MK, Swart AM; MRC OV05; EORTC 55955 Investigators: Early versus delayed treatment of relapsed ovarian cancer (MRC OV05/EORTC 55955): a randomised trial. Lancet 2010;376:1155-1163.

92 Medizinischer Dienst des Spitzenverbandes Bund der Krankenkassen e.V. (Hrsg): Begutachtungs-Richtlinie Vorsorge und Rehabilitation. Stand Februar 2012

93 Gemeinsamer Bundesausschuss: Richtlinie zur Regelung von Anforderungen an die Ausgestaltung strukturierter Behandlungsprogramme nach $₫ 137 \mathrm{f}$ Abs. 2 SGB V (DMP-Richtlinie/DMP-RL). Letzte Änderung: 20.11.2014. In Kraft getreten am: 07.01.2015.

94 Ibrahim EM, Abouelkhair KM, Kazkaz GA, Elmasri $\mathrm{OA}, \mathrm{Al}$-Foheidi M: Risk of second breast cancer in female Hodgkin's lymphoma survivors: a meta-analysis. BMC Cancer 2012;12:197.

95 Mulder RL, Kremer LC, Hudson MM, Bhatia S, Landier W, Levitt G, Constine LS, Wallace WH, van Leeuwen FE, Ronckers CM, Henderson TO, Dwyer M, Skinner R, Oeffinger KC; International Late Effects of Childhood Cancer Guideline Harmonization Group: Recommendations for breast cancer surveillance for female survivors of childhood, adolescent, and young adult cancer given chest radiation: a report from the International Late Effects of Childhood Cancer Guideline Harmonization Group. Lancet Oncol 2013;14:e621-629. 